\title{
Groups and analysis on fractals
}

\author{
Volodymyr Nekrashevych and Alexander Teplyaev
}

\begin{abstract}
We describe relation between analysis on fractals and the theory of self-similar groups. In particular, we focus on the construction of the Laplacian on limit sets of such groups in several concrete examples, and in the general p.c.f. case. We pose a number of open questions.
\end{abstract}

\section{Contents}

1. Introduction 1

2. Self-similar groups and their limit spaces 3

3. Representations of groups and functions $\quad 13$

4. Laplacians, Dirichlet forms and resistance forms 18

5. Examples of Laplacians on limit spaces 24

6. Analysis and probability on non p.c.f. fractals 33

References

\section{Introduction}

The aim of this survey is to show a new relation between two modern branches of fractal geometry: analysis on fractals and theory of self-similar groups. The reader can find background information, including detailed reference lists, in the books [Bar98, Kig01, Nek05, Str06], and throughout our paper we will mention a number of related articles.

One of the aims of analysis in general is construction of a natural Laplace operator, or Laplacian. There exists no strict, general definition of a Laplacian, but usually it is a local non-positive self-adjoint operator $\Delta$ on $L^{2}(\mathcal{X}, \nu)$ which is related to an energy form $\mathcal{E}$ by the relation

$$
\mathcal{E}(f, f)=-\int f \Delta f d \nu
$$

for an appropriately defined domain of functions $f$, and is the generator of the heat semigroup $P_{t}=e^{\Delta t}$. Having a Laplacian, one can consider the spectral problems, solutions to wave and heat equations, and many other objects and equations. If $\mathcal{E}$ is a Dirichlet form, which is defined in Subsection 4.1, then the Laplacian is the

2000 Mathematics Subject Classification. Primary:28A80, Secondary: 31C25, 20 E08.

Research supported in part by NSF grants DMS 0648786, DMS 0605019 and DMS 0505622, and by the Isaac Newton Institute for Mathematical Sciences. 
generator of a continuous diffusion process, and $P_{t}$ is its transition probability semigroup. If the underlaying set is compact and the measure is finite, then one often can expect some extra properties of the Laplacian, such as discrete spectrum and compactness of the resolvent. The general theory of self-adjoint operators and their semigroups and quadratic forms can be found in many books on functional analysis, mathematical physics, partial differential equations, and symmetric Markov processes, of which we mention [BH91, FŌT94, Lax02, RS80, Rud91]. Note that in some situations a Laplacian is defined as a non-negative operator, which corresponds to the absence of the minus sign in (1.1), but introduction of a minus sign in the definition of the semigroup $P_{t}$ of $\Delta$.

Theory of self-similar groups developed as a part of geometric group theory in recent decades. It was discovered that many exotic groups (like groups of intermediate growth, non-elementary amenable groups, infinite finitely generated torsion groups, etc.) can be easily defined and studied using their self-similar action on a regular rooted tree (see [Gri80, GS83, Gri85, BGŠ03]). The situation is quite analogous to fractal geometry, where historically first examples of fractals (like Sierpiński carpet, or Menger sponge) were exotic examples defined using their self-similar structure.

Recently a direct relation between self-similarity of groups and fractals via constructions of the iterated monodromy group and the limit space was discovered. These two constructions can be interpreted as mutually inverse functors between the properly defined categories of self-similar groups and expanding dynamical systems (and their Julia sets). See [Nek05, BGN03, Nek08a] and references therein for geometric aspects of the theory of self-similar groups.

For every contracting self-similar group $G$ there is a naturally defined limit space $\mathcal{J}_{G}$ together with an expanding branched self-covering s : $\mathcal{J}_{G} \longrightarrow \mathcal{J}_{G}$, see Definition 2.3. The group $G$ does not act on its limit space, but rather describes adjacency of cells of a natural self-similarity on $\mathcal{J}_{G}$, and can be interpreted as a kind of infinitesimal object related to the limit space (see, for instance Theorems 3.2 and 3.3 in our paper).

On the other hand, a self-similar group, called iterated monodromy group is associated with every partial self-covering $f: \mathcal{M}_{1} \longrightarrow \mathcal{M}$. As it was already mentioned, this construction is inverse to the construction of the limit space (under appropriate hyperbolicity conditions), which makes it possible to represent many well known fractals as limit spaces of contracting self-similar groups, thus endowing them with additional algebraic structure.

We hope that interaction between the theory of self-similar groups and analysis on fractals will be productive and useful for both branches of Mathematics. The following natural questions could be the first directions of research.

QuESTION 1. Is there a natural nontrivial Laplacian on the limit space $\mathcal{J}_{G}$ of a contracting group $G$ ? A natural Laplacian has to inherit in some sense the self-similarity of the group and its limit space.

It would be very nice to have some unique (up to a constant multiple) selfsimilar Laplacian on $\mathcal{J}_{G}$, but maybe it will be more realistic to ask for a family of self-similar Laplacians on $\mathcal{J}_{G}$. In any case, the following question is natural.

QUESTION 2. When is a self-similar Laplacian on $\mathcal{J}_{G}$ unique up to a constant multiple? 
Question 3. Do discrete Laplacians on the Schreier graphs $\Gamma_{n}$ of a contracting group $(G, \mathrm{X})$ converge, in some natural sense and with appropriate normalization, to a nontrivial Laplacian on $\mathcal{J}_{G}$ ? For the definition of the Schreier graphs $\Gamma_{n}$, see Subsection 2.4.

At present these questions are far from being answered in general. However, we think that limit spaces of contracting self-similar groups and the associated matrix recursions is a natural setting to study Laplacians on self-similar spaces such as Julia sets of expanding maps.

In Section 5 we analyze several examples of limit spaces of contracting selfsimilar groups: the circle, Sierpiński gasket, Basilica and Pillow. We show how known and new techniques can be applied to construct Laplacians on them. More specifically, for each example we show how the "classical" Laplacian could be constructed using "analysis on fractals" techniques, and also how it can be constructed using matrix calculations based on the theory of self-similar groups.

Furthermore, we show that in a special class of strictly p.c.f. groups the limit space is a p.c.f. self-similar set, so that the Laplacian can be constructed using known techniques (see Subsection 4.3), and relate these techniques with the matrix recursions used in the theory of self-similar groups. We hope that algebraic tools of self-similar groups can be useful for analysis on fractals.

Our paper is organized as follows. In Section 2 we introduce self-similar groups and their limit spaces. In Section 3 give detailed descriptions of representations of self-similar groups and function spaces. In Section 4 we describe basic results on existence and uniqueness of Laplacians, Dirichlet forms and resistance forms on p.c.f. fractals. In Section 5 we give detailed descriptions of several examples of limit sets of self-similar groups where the Laplacian can be constructed by various methods. In Section 6 we give references related to analysis and probability on non p.c.f. fractals, including Sierpiński carpets.

\section{Self-similar groups and their limit spaces}

2.1. Trees and words. Let $X$ be a finite set, called alphabet. The set of finite words over the alphabet $X$ is denoted $X^{*}$. The empty word is denoted $\varnothing$. The set $\mathrm{X}^{*}$ has a natural structure of a rooted tree with the root $\varnothing$ in which a word $v \in \mathbf{X}^{*}$ is connected by edges to the words of the form $v x$ for $x \in \mathrm{X}$.

The set of infinite words $\mathbf{X}^{\omega}=\left\{x_{1} x_{2} \ldots: x_{i} \in \mathbf{X}\right\}$ is identified with the boundary of the tree $X^{*}$. Namely, we introduce a topology on the set $X^{*} \sqcup X^{\omega}$ by the basis of open sets $v \mathrm{X}^{*} \cup v \mathrm{X}^{\omega}$, where $v \mathrm{X}^{*}$ and $v \mathrm{X}^{\omega}$ are the sets of (finite and infinite) words starting with a fixed word $v \in \mathbf{X}^{*}$. With respect to this topology $X^{\omega}$ is homeomorphic to the direct infinite power of the discrete set $X$, hence is homeomorphic to the Cantor set, and $X^{*}$ is a dense countable subset of $X^{*} \sqcup X^{\omega}$.

We will also consider the set $\mathbf{X}^{-\omega}=\left\{\ldots x_{2} x_{1}: x_{i} \in \mathrm{X}\right\}$ of left-infinite words. A natural topology on $X^{*} \sqcup X^{-\omega}$ is given by a basis of open sets consisting of the sets of the form $\mathbf{X}^{*} v \sqcup \mathbf{X}^{-\omega} v$, where $\mathbf{X}^{*} v$ and $\mathbf{X}^{-\omega} v$ are the sets of words ending by a given finite word $v$.

An automorphism of the rooted tree $\mathrm{X}^{*}$ is a permutation of $\mathrm{X}^{*}$ preserving adjacency of the vertices and fixing the root $\varnothing$ (actually, every automorphism of the tree $X^{*}$ fixes the root, since it is the only vertex of degree $|X|$, all the other vertices have degree $|X|+1)$. Every automorphism of $X^{*}$ preserves the levels, i.e., the sets $\mathrm{X}^{n}$ of words of a given length $n$. 
A group acting on $\mathrm{X}^{*}$ by automorphisms is said to be level-transitive if it acts transitively on the levels.

For more on rooted trees and their automorphisms see [Sid98, GNS00].

2.2. Self-similar groups. Here we review the basic definitions from theory of self-similar groups. For more see the paper [BGN03] and the book [Nek05].

Definition 2.1. A self-similar group $(G, \mathrm{X})$ is a group $G$ acting by automorphisms on the rooted tree $\mathrm{X}^{*}$ such that for every $g \in G$ and every $x \in \mathrm{X}$ there exist $h \in G$ and $y \in \mathrm{X}$ such that

$$
g(x w)=y h(w)
$$

for all $w \in \mathbf{X}^{*}$.

Note that in conditions of the definition we have $y=g(x)$.

Applying Definition 2.1 several times we see that if $(G, \mathrm{X})$ is a self-similar group, then for every finite word $v \in \mathrm{X}^{*}$ there exists $h \in G$ such that

$$
g(v w)=g(v) h(w)
$$

for all $w \in \mathrm{X}^{*}$. We will denote $h=\left.g\right|_{v}$ and call it section (or restriction) of $g$ at $v$. It is easy to see that sections have the following properties:

$$
\left.g\right|_{v_{1} v_{2}}=\left.\left.g\right|_{v_{1}}\right|_{v_{2}},\left.\quad\left(g_{1} g_{2}\right)\right|_{v}=\left.\left.g_{1}\right|_{g_{2}(v)} g_{2}\right|_{v} .
$$

EXAMPLE 1. One of the most classical examples of a self-similar group is the binary adding machine action of the infinite cyclic group $\mathbb{Z}$. It is generated by one automorphism $a$ of the binary tree $X^{*}=\{0,1\}^{*}$, which is defined recursively by the rule

for all $w \in \mathbf{X}^{*}$.

$$
a(0 w)=1 w, \quad a(1 w)=0 a(w)
$$

The transformation $a$ (also called the binary adding machine) describes adding 1 to a binary integer. More explicitly, $a\left(x_{1} x_{2} \ldots x_{n}\right)=y_{1} y_{2} \ldots y_{n}$ if and only if

$$
\left(x_{1}+x_{2} 2+x_{3} 4+\cdots x_{n} 2^{n-1}\right)+1=y_{1}+y_{2} 2+y_{3} 4+\cdots y_{n} 2^{n-1} \quad\left(\bmod 2^{n}\right) .
$$

EXAmple 2. Consider the group generated by two automorphisms $a$ and $b$ of the binary rooted tree, which are defined by the rules

$$
\begin{array}{ll}
a(0 w)=1 w, & a(1 w)=0 b(w), \\
b(0 w)=0 w, & b(1 w)=1 a(w) .
\end{array}
$$

The group generated by $a$ and $b$ is the iterated monodromy of the complex polynomial $z^{2}-1$. It is closely related to the combinatorics of iterations of this polynomial

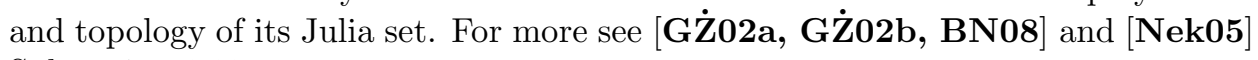
Subsection 6.12.1.

EXAMPLE 3. The group generated by three automorphisms of the binary tree given by

$$
\begin{array}{llrl}
a(0 w) & =1 b(w), & & a(1 w)=0 c(w), \\
b(0 w) & =0 a(w), & & b(1 w)=1 a(w), \\
c(0 w) & =1 c(w), & & c(1 w)=0 b(w)
\end{array}
$$

is isomorphic to the free group freely generated by $a, b$ and $c$, see [VV07]. 


\subsection{Contracting self-similar groups and their limit spaces.}

Definition 2.2. A self-similar group $G$ is said to be contracting if there exists a finite set $\mathcal{N} \subset G$ such that for every $g \in G$ there exists $n$ such that $\left.g\right|_{v} \in \mathcal{N}$ for all words $v$ of length more than $n$. The smallest set $\mathcal{N}$ with this property is called the nucleus of the contracting group.

EXAMPLE 4 . It is easy to check that the binary adding machine action, described in Example 1 is contracting with the nucleus $\left\{a^{-1}, a^{0}, a\right\}$.

EXAMPLE 5. The iterated monodromy group of $z^{2}-1$ described in Example 2 is contracting with the nucleus $\left\{1, a, a^{-1}, b, b^{-1}, a b^{-1}, b a^{-1}\right\}$. If a self-similar group $G$ is generated by a finite set $S$, then a finite set $\mathcal{N} \subset G$ such that $1 \in \mathcal{N}$ and $\left.g\right|_{x} \in \mathcal{N}$ for all $g \in \mathcal{N}$ and $x \in \mathrm{X}$, contains the nucleus of $G$ if and only if there exists $n \in \mathbb{N}$ such that $\left.(g s)\right|_{v} \in \mathcal{N}$ for all $g \in \mathcal{N}, s \in S \cup S^{-1} \cup\{1\}$ and $v \in \mathbf{X}^{n}$. Checking this condition for given $n, S$ and $\mathcal{N}$ is a finite computation.

The free group from Example 3 is not contracting. Moreover, it is proved in [Nek07] that contracting groups have no free subgroups.

A more geometric characterization of contracting self-similar group is given in the following proposition (for its proof see Lemma 2.11.10 and Proposition 2.11.11 of $[\mathrm{Nek05}])$.

Proposition 2.1. Let $(G, \mathrm{X})$ be a finitely generated self-similar group and denote by $l(g)$ length of an element $g \in G$ with respect to a fixed finite generating set $S$ of $G$ (i.e., $l(g)$ is length of the shortest representation of $g$ as a product of elements of $S$ and their inverses).

Then the number

$$
\rho=\limsup _{n \rightarrow \infty} \sqrt[n]{\max _{v \in \mathrm{X}^{n}} \limsup _{l(g) \rightarrow \infty} \frac{l\left(\left.g\right|_{v}\right)}{l(g)}}
$$

is finite, does not depend on the choice of the generating set $S$. It is less than one if and only if the action is contracting.

We call $\rho$ the contraction coefficient of the self-similar action. For more on algebraic and geometric properties of contracting self-similar groups see [Nek05].

Definition 2.3. Let $(G, \mathrm{X})$ be a contracting self-similar group. Two leftinfinite sequences $\ldots x_{2} x_{1}, \ldots y_{2} y_{1} \in \mathbf{X}^{-\omega}$ are said to be (asymptotically) equivalent if there exists a finite set $N \subset G$ and a sequence $g_{k} \in N$ for $k=1,2, \ldots$ such that

$$
g_{k}\left(x_{k} \ldots x_{2} x_{1}\right)=y_{k} \ldots y_{2} y_{1}
$$

for all $k \geq 1$.

The quotient $\mathcal{J}_{G}$ of the topological space $\mathbf{X}^{-\omega}$ of left-infinite sequences by the asymptotic equivalence relation is called the limit space of the self-similar group $G$.

It is easy to see that the asymptotic equivalence relation is invariant under the shift $\ldots x_{2} x_{1} \mapsto \ldots x_{3} x_{2}$, hence the shift induces a continuous self-map of $\mathcal{J}_{G}$, which we will denote s. The dynamical system $\left(\mathcal{J}_{G}, \mathrm{~s}\right)$ is called the limit dynamical system of the contracting group $G$.

The following description of the asymptotic equivalence relation is proved in Theorem 3.6.3 of [Nek05] . 


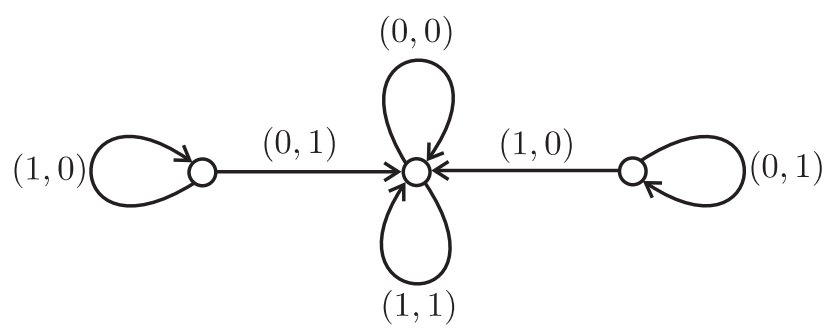

Figure 1. Nucleus of the adding machine action

Proposition 2.2. Let $\mathcal{N}$ be the nucleus of a contracting group $(G, \mathrm{X})$. Two sequences $\ldots x_{2} x_{1}, \ldots y_{2} y_{1} \in \mathbf{X}^{-\omega}$ are equivalent with respect to the action of $G$ if and only if there exists a sequence $g_{k} \in \mathcal{N}, k=0,1, \ldots$, such that $g_{k}\left(x_{k}\right)=y_{k}$ and $\left.g_{k}\right|_{x_{k}}=g_{k-1}$ for all $k=1,2, \ldots$.

Consider a directed labeled graph with the set of vertices $\mathcal{N}$ in which for every $g \in \mathcal{N}$ and every $x \in \mathrm{X}$ we have an arrow starting in $g$, ending in $\left.g\right|_{x}$ and labeled by $(x, g(x))$. This graph is called the Moore diagram of the nucleus. Then the previous proposition says that two sequences $\ldots x_{2} x_{1}, \ldots y_{2} y_{1} \in \mathbf{X}^{-\omega}$ are equivalent if and only if there exists an oriented left-infinite path $\ldots e_{2} e_{1}$ of arrows such that for every $k$ the arrow $e_{k}$ is labeled by $\left(x_{k}, y_{k}\right)$.

EXAMPLE 6 . The Moore diagram of the nucleus of the adding machine action is shown on Figure 1.

One can see from the diagram that two sequences are asymptotically equivalent if and only if they are either equal or of the form $\ldots 0001 v, \ldots 1110 v$ for some finite word $v$, or they are ...000 and ...111. But this is the usual identification of the binary reals, so that $\ldots x_{2} x_{1}, \ldots y_{2} y_{1} \in \mathbf{X}^{-\omega}$ are equivalent with respect to the adding machine action if and only if

$$
\sum_{n \geq 1} x_{n} 2^{-n}=\sum_{n \geq 1} y_{n} 2^{-n} \quad(\bmod 1) .
$$

It follows that the limit space of this self-similar group is the circle $\mathbb{R} / \mathbb{Z}$, where encoding of its points by sequences comes from the binary numeration system on the reals.

In particular, the shift $\mathbf{s}$ on the limit space is the double covering map $x \mapsto 2 x$ $(\bmod 1)$ of $\mathbb{R} / \mathbb{Z}$.

We will give more examples of the limit spaces of contracting groups later.

The following properties of the limit space $\mathcal{J}_{G}$ are proved in [Nek05], Theorem 3.6.3.

Definition 2.4. A self-similar group $G$ is called self-replicating (or recurrent in [Nek05]) if for every pair of letters $x, y \in \mathbf{X}$ and every $h \in G$ there exists $g \in G$ such that $g(x)=y$ and $\left.g\right|_{x}=h$.

Proposition 2.3. The limit space $\mathcal{J}_{G}$ is compact, metrizable and has finite topological dimension. It is connected if the group $G$ is level-transitive. If the group $G$ is self-replicating, then the limit space $\mathcal{J}_{G}$ is locally connected and path connected.

Let $(G, \mathrm{X})$ be a contracting self-similar group. Let $S \subset G$ be a finite generating set of $G$ and suppose that it is state-closed, i.e., that for every $s \in S$ and every 


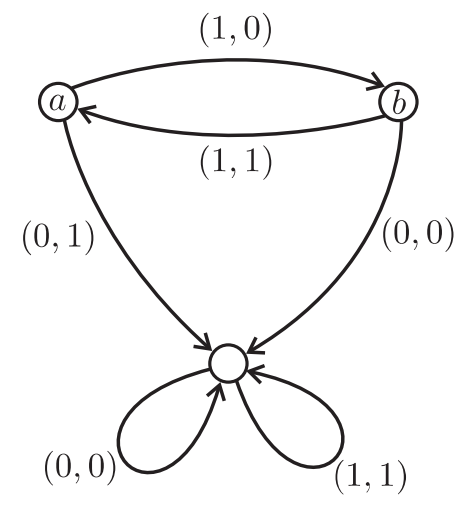

Figure 2. Generating set of IMG $\left(z^{2}-1\right)$

$x \in \mathrm{X}$ the section $\left.s\right|_{x}$ also belongs to $S$. If $G$ is finitely generated and contracting, then every finite generating set of $G$ is a subset of a state-closed finite generating set. The Moore diagram of the set $S$ is defined in the same way as the Moore diagram of the nucleus: it is the graph with the set of vertices $S$ with the same agreement on the arrows and their labeling.

Then the asymptotic equivalence relation on $\mathbf{X}^{-\omega}$ can be also described in the following way, see a proof in [Nek05], Proposition 3.6.4.

Proposition 2.4. Let $(G, \mathrm{X})$ be a contracting self-similar group and let $S$ be its finite state-closed generating set. Let $D$ be the set of pairs $\left(\ldots x_{2} x_{1}, \ldots y_{2} y_{1}\right) \in$ $\mathbf{X}^{-\omega} \times \mathbf{X}^{-\omega}$, such that there exists a left-infinite oriented path $\ldots e_{2} e_{1}$ in the Moore diagram of $S$ in which every arrow $e_{i}$ is labeled by $\left(x_{i}, y_{i}\right)$.

Then the asymptotic equivalence on $\mathrm{X}^{-\omega}$ associated with the group $(G, \mathrm{X})$ is the equivalence generated by the set $D$.

ExAmple 7. The Moore diagram of the generating set $\{1, a, b\}$ of the iterated monodromy group of $z^{2}-1$ is shown on Figure 2 .

We see that the associated asymptotic equivalence relation is generated by the identifications

$$
\begin{array}{ll}
(11)^{-\omega} 0 v=(01)^{-\omega} 1 v, & (11)^{-\omega}=(01)^{-\omega}, \\
(11)^{-\omega} 0 v=(10)^{-\omega} 0 v, & (11)^{-\omega}=(10)^{-\omega} .
\end{array}
$$

2.4. Schreier graphs and tiles. Let $G$ be a group acting on a set $M$ and let $S$ be a finite generating set of $G$. Then the associated Schreier graph, or graph of the action $\Gamma(G, M, S)$ is the directed graph with the set of vertices $M$ with the set of arrows $S \times M$, where the arrow $(s, x)$ starts at $x$ and ends in $s(x)$. We label the arrow $(s, x)$ by $s$. In this way the Schreier graph uniquely defines the action of $G$ on $M$.

The associated simplicial Schreier graph is the graph with the set of vertices $M$ in which two different vertices are connected by an edge if and only if one is the image of the other under the action of a generator $s \in S$. In other words, we erase in the simplicial Schreier graph all the information about orientation, labeling, multiple edges, loops, and leave only the adjacency relation on the set of vertices. 


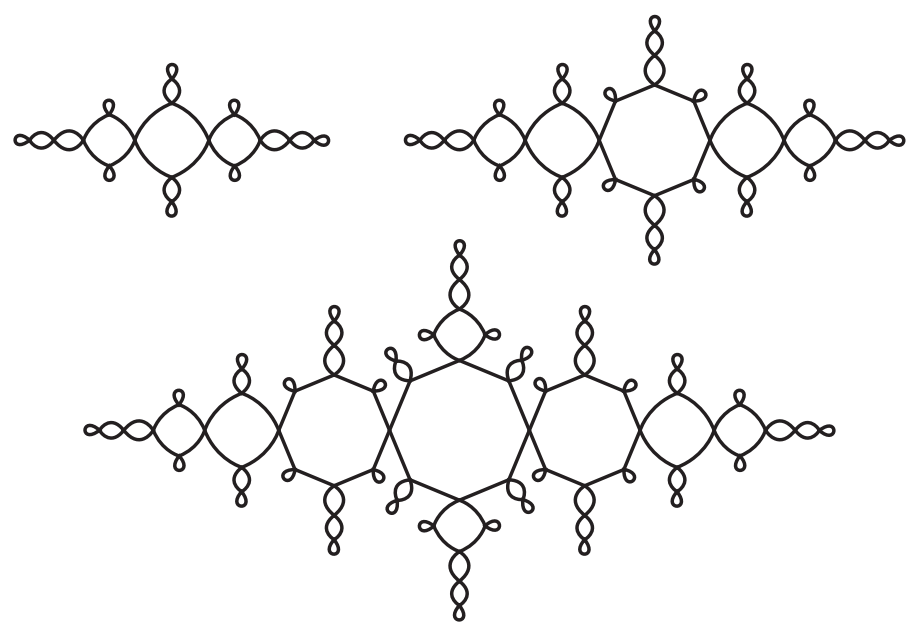

FIGURE 3. Schreier graphs of IMG $\left(z^{2}-1\right)$

If $G$ is a finitely generated group acting on a rooted tree $\mathrm{X}^{*}$, then we naturally get the Schreier graphs $\Gamma_{n}=\Gamma\left(G, \mathrm{X}^{n}, S\right)$ of the action of $G$ on the levels of the tree.

For instance, the Schreier graphs $\Gamma_{n}$ of the action of the adding machine on the $n$th level of the binary tree is a cycle of length $2^{n}$ (with respect to the generating set $\{a\}$.

The Schreier graphs of the action of the iterated monodromy group of $z^{2}-1$ on the levels $X^{4}, X^{5}$ and $X^{6}$ are shown on Figure 3.

Approximation of the limit space by the Schreier graphs is a general fact, which can be formalized in different ways. One of the formalizations uses the notion of a Gromov hyperbolic graph and is contained in the following theorem.

THEOREM 2.5. Let $(G, \mathrm{X})$ be a contracting self-similar group generated by a finite set $S$. Consider the graph $\Sigma$ with the set of vertices $\mathrm{X}^{*}$ in which two vertices are connected by an edge in the following two cases:

(1) if they are of the form $v$ and $x v$, where $v \in \mathrm{X}^{*}$ and $x \in \mathrm{X}$ (vertical edges);

(2) if they are of the form $v$ and $s(v)$, where $v \in \mathrm{X}^{*}$ and $s \in S$ (horizontal edges).

Then the graph $\Sigma$ is Gromov hyperbolic and its hyperbolic boundary is homeomorphic to the limit space $\mathcal{J}_{G}$. GH90].

For a definition of Gromov hyperbolicity and hyperbolic boundary see [Gro87,

Note that the horizontal edges form a graph isomorphic to the disjoint union of the simplicial Schreier graphs of the action of $G$ on the levels of the tree $\mathrm{X}^{*}$. The vertical edges form a tree isomorphic to $\mathrm{X}^{*}$ (though the isomorphism is not the identity map, but the map inverting the order of the letters in the words).

Representation of the limit space $\mathcal{J}_{G}$ as a boundary of a hyperbolic graph can be used to introduce a quasi-symmetric structure on $\mathcal{J}_{G}$. See [HP06] for more details in this and in a more general setting. 
Another formalization of the relation between the Schreier graphs and the limit space uses the notion of a tile of the limit space.

Definition 2.5. Let $(G, \mathrm{X})$ be a contracting group. A tile of the $n$th level $\mathcal{T}_{v}$ is the image in $\mathcal{J}_{G}$ of the set $\mathrm{X}^{-\omega} v$ of sequences ending by $v$.

It is easy to see that the following properties of tiles hold.

$$
\mathcal{T}_{v}=\bigcup_{x \in \mathrm{X}} \mathcal{T}_{x v}, \quad \mathrm{~s}\left(\mathcal{T}_{v x}\right)=\mathcal{T}_{v}
$$

where $x \in \mathbf{X}$ and $v \in \mathbf{X}^{*}$ are arbitrary.

EXAMPLE 8. In the case of the binary adding machine action (Example 1 and its limit space $\mathbb{R} / \mathbb{Z}$ (see Example 6 ) a tile $\mathcal{T}_{v}$ for $v=x_{n} \ldots x_{1}$ for $n \geq 1$ is the interval $\left[p, p+\frac{1}{2^{n}}\right]$, where $p=x_{1} / 2+x_{2} / 4+\cdots+x_{n} / 2^{k}$. We see that the adjacency graph of the tiles of $n$th level is a cycle of $2^{n}$ points, which agrees with the fact that the adding machine $a$ acts on the $n$th level of the tree $\mathrm{X}^{*}$ as a transitive cycle.

The following properties of tiles are proved in [Nek05], Propositions 3.6.5 and 3.6.8.

Proposition 2.6. If for every $g \in G$ there exists $v \in \mathrm{X}^{*}$ such that $\left.g\right|_{v}=1$, then the tiles are equal to the closures of their interiors, two tiles of the same level have disjoint interiors; and the boundary of a tile $\mathcal{T}_{v} \subseteq \mathcal{J}_{G}$ is equal to the set of points of $\mathcal{T}_{v}$ belonging to the other tiles of the same level.

We will say that a self-similar group $(G, \mathrm{X})$ satisfies the open set condition if for every $g \in G$ there exists $v \in \mathrm{X}^{*}$ such that $\left.g\right|_{v}=1$. It is not hard to see that it is sufficient to check this condition only for the elements of a generating set of $G$.

Proposition 2.7. Let $\mathcal{N}$ be the nucleus of the group $G$. Then two tiles $\mathcal{T}_{v}$ and $\mathcal{T}_{u}$ of the same level intersect if and only if there exists $g \in \mathcal{N}$ such that $g(v)=u$.

The following proposition describes the boundary of a tile $\mathcal{T}_{v}$, which will be very convenient in the case when the boundary is finite (in the case of groups of bounded automorphisms). Its proof can be found in [BN03] and [Nek05] (Theorem 3.9.12).

Proposition 2.8. Suppose that a contracting group $(G, \mathrm{X})$ satisfies the open set condition. Then the boundary of a tile $\mathcal{T}_{v}$ consists of the points, which can be represented by sequences ... $x_{2} x_{1} \in \mathrm{X}^{-\omega}$ such that there exists a sequence $\left\{g_{k}\right\}_{k \geq 0}$ of elements of the nucleus of $G$ such that $\left.g_{k}\right|_{x_{k}}=g_{k-1}$ and $g_{0}(v) \neq v$.

If a self-similar group $(G, \mathrm{X})$ is self-replicating and finitely generated, then it is generated by its nucleus. Consequently, the tiles of the $n$th level of $\mathcal{J}_{G}$ are adjacent in the same way as the vertices of the Schreier graph $\Gamma\left(G, X^{n}, \mathcal{N}\right)$ are adjacent. The tiles of higher levels subdivide the tiles of the previous levels due to (2.2). More formally, we have the following description of the limit space $\mathcal{J}_{G}$ (see [Nek05], Theorem 3.6.9).

TheOREM 2.9. A compact Hausdorff space $\mathcal{X}$ is homeomorphic to the limit space $\mathcal{J}_{G}$ if and only if there exists a collection $\mathfrak{T}=\left\{T_{v}: v \in \mathrm{X}^{*}\right\}$ of closed subsets of $\mathcal{X}$ such that the following conditions hold.

(1) $T_{\varnothing}=\mathcal{X}$ and $T_{v}=\bigcup_{x \in \mathrm{X}} T_{x v}$ for every $v \in \mathrm{X}^{*}$.

(2) The set $\bigcap_{n=1}^{\infty} T_{x_{n} x_{n-1} \ldots x_{1}}$ contains only one point for every word $\ldots x_{2} x_{1} \in$ $\mathrm{X}^{-\omega}$. 
(3) The intersection $T_{v} \cap T_{u}$ for $u, v \in \mathrm{X}^{n}$ is non-empty if and only if there exists an element $s$ of the nucleus such that $s(v)=u$.

2.5. Groups generated by bounded automata. We say that an automorphism $g$ of $\mathbf{X}^{*}$ is bounded if the Moore diagram of the set $\left\{\left.g\right|_{v}: v \in \mathbf{X}^{*}\right\}$ is finite and its oriented cycles consisting of non-trivial elements are disjoint and not connected by directed paths. The notion of a bounded automorphism of $\mathrm{X}^{*}$ was defined and studied for the first time by S. Sidki in [Sid00].

An automorphism $g$ of the tree $\mathbf{X}^{*}$ is said to be finitary if there exists $n \in \mathbb{N}$ such that $g(v w)=g(v) w$ for all $v \in \mathbf{X}^{n}$ and $w \in \mathbf{X}^{*}$, i.e., if $g$ changes in every word only beginnings of length $n$.

An automorphism $g$ of $\mathrm{X}^{*}$ is said to be directed if there exists $n \in \mathbb{N}$ and $v \in \mathrm{X}^{n}$ such that $\left.g\right|_{v}=g$ and $\left.g\right|_{u}$ is finitary for all $u \in \mathbf{X}^{n} \backslash\{v\}$.

The following description of bounded automorphisms is more or less straightforward.

Proposition 2.10. An automorphism $g$ of the tree $\mathrm{X}^{*}$ is bounded if and only if there exists $n \in \mathbb{N}$ such that $\left.g\right|_{v}$ is either finitary or directed for all $v \in \mathbf{X}^{n}$.

The set $\mathcal{B}(\mathrm{X})$ of all bounded automorphisms of $\mathrm{X}^{*}$ is a group.

The following description of self-similar subgroups of $\mathcal{B}(X)$ is given in [BN03].

THEOREM 2.11. Every self-similar subgroup of the group of bounded automata $\mathcal{B}(\mathrm{X})$ is contracting.

A contracting self-similar group is a subgroup of $\mathcal{B}(\mathrm{X})$ if and only if it satisfies the open set condition and the boundaries of the tiles $\mathcal{T}_{v}$ are finite for all $v \in \mathrm{X}^{*}$.

In particular, the limit space of a self-similar subgroup of $\mathcal{B}(\mathrm{X})$ is topologically one-dimensional. The limit spaces of self-similar groups of bounded automorphisms can be visualized using a simple iterative procedure described in [Nek05] Section 3.10.

Subgroups of $\mathcal{B}(\mathrm{X})$ are the most well-studied examples of self-similar groups. Most of the well-known examples of contracting self-similar groups (such as the Grigorchuk group [Gri80], Gupta-Sidki group [GS83], the group from [BSV99], etc.) are subgroups of the group of bounded automorphisms of the respective rooted tree $X^{*}$. All iterated monodromy groups of polynomials are generated by bounded automorphisms, see [Nek05] (Sections 6.7-6.10, in particular Theorem 6.10.8).

A study of random walks on groups generated by bounded automorphisms produced the following result, proved in [BKN08].

TheOREM 2.12. The group $\mathcal{B}(\mathrm{X})$ of bounded automorphisms of the tree $\mathrm{X}^{*}$ is amenable.

2.6. Iterated monodromy groups. Self-similar groups appeared historically as interesting examples of groups with exotic properties (see [Gri80, GS83]). It was discovered later that they have natural connections to dynamical systems and fractals via the constructions of the limit space and the limit dynamical system. A more direct relation was established when the theory of the iterated monodromy group was developed (see [Nek03, BGN03] and [Nek05]). Iterated monodromy groups became an important source of interesting self-similar groups and the technique of self-similar groups started to be used to solve problems in dynamical systems (see, for instance, [BN06]). 
Let $\mathcal{M}$ be a path connected and locally path connected topological space. A partial self-covering of $\mathcal{M}$ is a covering map $f: \mathcal{M}_{1} \longrightarrow \mathcal{M}$ from a subset $\mathcal{M}_{1} \subset$ $\mathcal{M}$ onto $\mathcal{M}$. Here a map is called a covering map if every point $x \in \mathcal{M}$ has a neighborhood $U$ such that $f^{-1}(U)$ is a disjoint union of subsets $U_{i}$ such that $f: U_{i} \longrightarrow U$ is a homeomorphism. The cardinality $\left|f^{-1}(x)\right|$ of a fiber is called the degree of the covering (it does not depend on the choice of a point $x \in \mathcal{M}$, since $\mathcal{M}$ is connected). A covering is called $d$-fold if its degree is $d$.

Definition 2.6. Let $f: \mathcal{M}_{1} \longrightarrow \mathcal{M}$ be a partial self-covering. Denote by $f^{n}: \mathcal{M}_{n} \longrightarrow \mathcal{M}$ the $n$th iteration of $f$ (we have $\mathcal{M}_{n} \subseteq \mathcal{M}_{n-1}$ ) and choose a basepoint $t \in \mathcal{M}$. The tree of preimages is the rooted tree with the set of vertices equal to the formal disjoint union

$$
T_{t}=\bigsqcup_{n \geq 0} f^{-n}(t)
$$

of the inverse images of $t$ under the iterations of $f$ (here $f^{-0}(t)=\{t\}$ ), where every vertex $z \in f^{-n}(t)$ is connected to the vertex $f(z) \in f^{-(n-1)}(t)$. The vertex $t \in f^{-0}(t)$ is the root of the tree $T_{t}$.

The iterated monodromy action of the fundamental group $\pi_{1}(\mathcal{M}, t)$ on the tree $T_{t}$ is the natural action of the fundamental group on the fibers $f^{-n}(t)$ of the coverings $f^{n}$. Namely, the image of a point $z \in f^{-n}(t)$ under the action of a loop $\gamma \in \pi_{1}(\mathcal{M}, t)$ is the endpoint of the unique lift of $\gamma$ by $f^{n}$ which starts at $z$.

It is easy to see that the iterated monodromy action is an action by automorphisms of the tree $T_{t}$.

Definition 2.7. The iterated monodromy group IMG $(f)$ of a partial selfcovering $f: \mathcal{M}_{1} \longrightarrow \mathcal{M}$ is the quotient of the fundamental group $\pi_{1}(\mathcal{M}, t)$ by the kernel of the iterated monodromy action. In other words, it is the group of the automorphisms of the tree of preimages defined by the iterated monodromy actions of the elements of the fundamental group.

It is not hard to show that the iterated monodromy group does not depend on the choice of the basepoint $t \in \mathcal{M}$.

The action of the iterated monodromy group on the tree of preimages is a selfsimilar group, if we label the vertices of the tree $T_{t}$ by words over an alphabet $\mathrm{X}$, $|\mathrm{X}|=\operatorname{deg} f$, in an appropriate way. Namely, the following proposition can be used to compute iterated monodromy groups by their standard self-similar action.

Proposition 2.13. Let $f: \mathcal{M}_{1} \longrightarrow \mathcal{M}$ be a partial self-covering of degree $d$. Let $\mathrm{X}$ be any alphabet of size $d$. Choose a basepoint $t \in \mathcal{M}$, a bijection $L: \mathrm{X} \longrightarrow$ $f^{-1}(t)$ and for every $x \in \mathbf{X}$ a path $\ell_{x}$ starting at $t$ and ending in $L(x)$.

Define then the associated standard self-similar action of the fundamental group $\pi_{1}(\mathcal{M}, t)$ on $\mathrm{X}^{*}$ by the rule

$$
\gamma(x v)=y\left(\ell_{x} \gamma_{x} \ell_{y}^{-1}\right)(v),
$$

where $\gamma_{x}$ is the $f$-lift of $\gamma$ starting in $L(x), y \in \mathbf{X}$ is such that $L(y)$ is the end of $\gamma_{x}$ and $v \in \mathrm{X}^{*}$ is arbitrary.

Then the standard self-similar action is conjugate with the iterated monodromy action of $\pi_{1}(\mathcal{M}, t)$ on $T_{t}$. In particular, the self-similar group defined by the recursion (2.3) is isomorphic to the iterated monodromy group of $f$. 


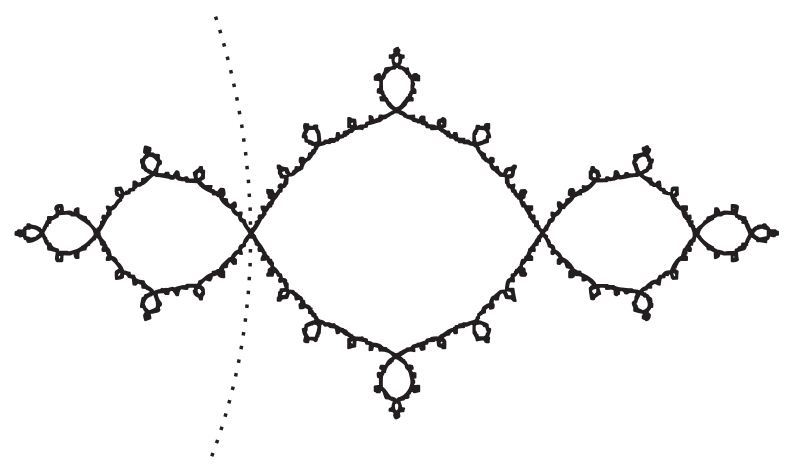

Figure 4. The Julia set of $z^{2}-1$

For examples of computation of iterated monodromy groups see [BGN03, Nek05].

Suppose that $f: \mathcal{M}_{1} \longrightarrow \mathcal{M}$ is expanding, i.e., that $\mathcal{M}$ is a complete Riemannian manifold and there exist $C>0$ and $\lambda>1$ such that the differential $D f^{\circ n}$ expands the length of every non-zero tangent vector to $f^{-n}(\mathcal{M}) \subset \mathcal{M}$ by a factor of at least $C \lambda^{n}$, where $f^{-n}(\mathcal{M})=\mathcal{M}_{n}$ is considered with the induced metric.

Define the Julia set of $f$ as the set of accumulation points of the backward orbit $\bigcup_{n>0} f^{-n}(t)$. Note that it is not the classical definition of the Julia set, but it coincides with the classical one in the case of a post-critically finite complex rational function (in the one-dimensional case).

Theorem 2.14. Let $f: \mathcal{M}_{1} \longrightarrow \mathcal{M}$ be an expanding partial self-covering and let $L: \mathrm{X} \longrightarrow f^{-1}(t)$ and $\ell_{x}$ be as in Proposition 2.13. Then the associated standard self-similar action of IMG $(f)$ is contracting and the limit dynamical system $\left(\mathcal{J}_{\operatorname{IMG}(f)}, \mathrm{s}\right)$ is topologically conjugate with the restriction of $f$ on its Julia set $J_{f}$.

For example, compare the Julia set of $z^{2}-1$ shown on Figure 4 (the dotted line is not part of the Julia set) with the Schreier graphs of its iterated monodromy group, shown on Figure 3.

Moreover, the conjugating homeomorphism $L: \mathcal{J}_{G} \longrightarrow J_{f}$ can be described in the following way. If $\zeta \in \mathcal{J}_{G}$ is represented by a sequence $\ldots x_{2} x_{1} \in \mathrm{X}^{-\omega}$, then $L(\zeta)$ is the limit (endpoint) of the path

$$
\ell_{1} \ell_{2} \ell_{3} \ldots
$$

where $\ell_{n}$ is defined inductively by the conditions $\ell_{1}=\ell_{x_{1}}$ and that $\ell_{n}$ is the lift of $\ell_{x_{n}}$ by $f^{n}$ starting at the endpoint of $\ell_{n-1}$.

\subsection{Some examples of limit spaces of contracting groups.}

2.7.1. Julia sets of rational functions. A rational function $f \in \mathbb{C}(z)$ is said to be sub-hyperbolic if orbit of every critical point of $f$ is either finite, or is converging to an attracting cycle. Sub-hyperbolic functions are expanding on a neighborhood of their Julia set with respect to some orbifold metric (see [Mil99]). This implies (by a version of Theorem 2.14 for orbifolds, see [Nek05] Theorem 5.5.3.) the following realization of the Julia sets as limit spaces.

THEOREM 2.15. The iterated monodromy group of a sub-hyperbolic rational function $f$ is contracting with respect to any standard self-similar action (IMG $(f), \mathrm{X})$. 


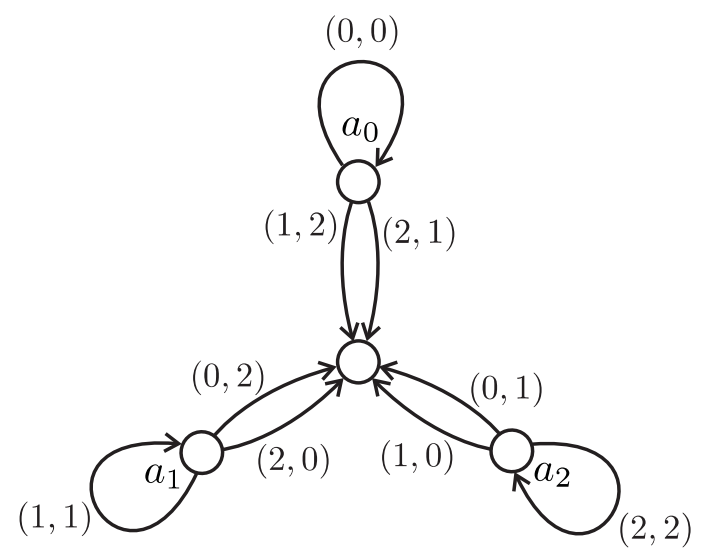

FiguRE 5. Moore diagram of $H_{3}$

Moreover, restriction of $f$ onto its Julia set is topologically conjugate with the limit dynamical system $\left(\mathcal{J}_{\operatorname{IMG}(f)}, \mathrm{s}\right)$.

Iterated monodromy groups of sub-hyperbolic polynomials are described in Sections 6.7-6.10 of [Nek05]. In particular, it is shown there that iterated monodromy groups of sub-hyperbolic polynomials are subgroups of the group $\mathcal{B}(\mathrm{X})$ of bounded automorphisms of $\mathrm{X}^{*}$.

2.7.2. Hanoi tower group. Consider the self-similar group $H_{3}$ over the alphabet $\{0,1,2\}$ generated by the transformations $a_{0}, a_{1}, a_{2}$ given by the recurrent rules

$$
\begin{array}{lll}
a_{0}(0 w)=0 a_{0}(w), & a_{0}(1 w)=2 w, & a_{0}(2 w)=1 w, \\
a_{1}(0 w)=2 w, & a_{1}(1 w)=1 a_{1}(w), & a_{1}(2 w)=0 w, \\
a_{2}(0 w)=1 w, & a_{2}(1 w)=0 w, & a_{2}(2 w)=2 a_{2}(w) .
\end{array}
$$

This group models the well known "Hanoi tower" game. In this model words of length $n$ over the alphabet $\{0,1,2\}$ describe configurations of the game (with $n$ discs) in a natural way, and the three generators correspond to three possible moves applied to a configuration. See more details in [GŠ06, GŠ08].

The Moore diagram of the set $\left\{1, a_{0}, a_{1}, a_{2}\right\}$ is shown on Figure 5 . The trivial state is drawn in the center of the figure without the obvious loops attached to it.

Tracking the paths in the Moore diagram, we see that two elements of $\mathbf{X}^{-\omega}$ are identified in the limit space $\mathcal{J}_{H_{3}}$ if and only if they are either equal or of the form $x^{-\omega} y v$ and $x^{-\omega} z v$, where $\{x, y, z\}=\{0,1,2\}$ and $v \in \mathrm{X}^{*}$. It is easy to deduce from this that the limit space is homeomorphic to the Sierpiński gasket, shown on the left-hand side of Figure 6 . The right-hand side of Figure 6 shows the Schreier graphs of the action of the group $H_{3}$ on the fourth level of the tree $\mathbf{X}^{*}$. It is thus the graph of the Hanoi tower game with four discs. Note that, in accordance with Proposition 2.7, the Schreier graph coincides with the adjacency graph of the tiles of the Sierpiński gasket of the corresponding level.

\section{Representations of groups and functions}

3.1. Matrix recursions. Let $(G, \mathrm{X})$ be a self-similar group. Let us order the letters of the alphabet $\mathbf{X}$, identifying it with $\{0,1, \ldots, d-1\}$. 

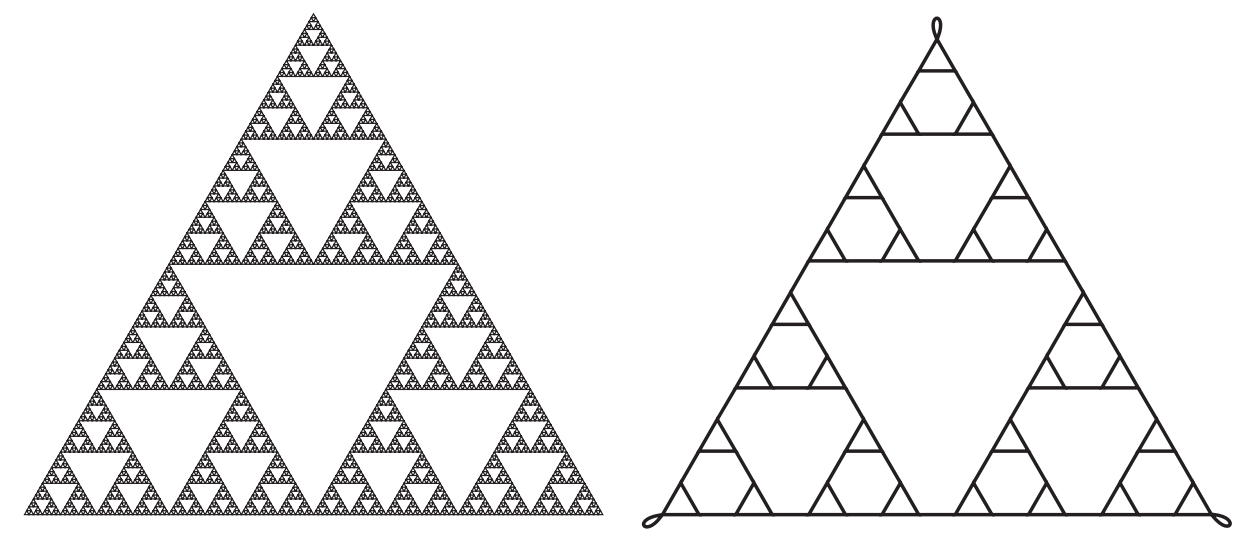

Figure 6. The limit space and a Schreier graph of $H_{3}$

Every element $g \in G$ induces a permutation $x \mapsto g(x)$ of the alphabet $\mathrm{X}$ and defines a sequence of group elements $\left.g\right|_{i}$, for $i \in \mathrm{X}$. We can encode this information as a matrix $\psi(g)=\left(a_{i j}\right)_{1 \leq i, j \leq d}$ of elements of the subset $G \sqcup\{0\}$ of the group ring $\mathbb{C} G$, defined by the rule

$$
a_{i j}= \begin{cases}\left.g\right|_{j} & \text { if } g(j)=i, \\ 0 & \text { otherwise. }\end{cases}
$$

Every such a matrix belongs to the set $M_{G}$ of matrices, which are obtained from a permutational matrix by replacing its non-zero entries by elements of $G$. The set of matrices $M_{G}$ is naturally isomorphic to the wreath product $S_{d} \ltimes G^{d}$ of $G$ with the symmetric group $S_{d}$.

In this way we get a map $\psi$ from $G$ to the algebra of $d \times d$-matrices over the group ring $\mathbb{C} G$. It follows directly from (2.1) on page 4 that the map $\psi$ is a homomorphism of the group $G$ to a subgroup of $M_{G} \subset M_{d \times d}(\mathbb{C} G)$.

The map $\psi$ is called the matrix recursion associated with the self-similar group $(G, \mathrm{X})$. We can extend it by linearity to a homomorphism $\psi: \mathbb{C} G \longrightarrow M_{d \times d}(\mathbb{C} G)$. This extension is also called the matrix recursion.

The map $\psi$ as a homomorphism of the group $G$ into the wreath product $S_{d} \ltimes G^{d}$ is called wreath recursion of the self-similar group. The wreath recursion is an embedding, unlike the matrix recursion $\psi: \mathbb{C} G \longrightarrow M_{d \times d}(\mathbb{C} G)$.

Definition 3.1. Let $(G, \mathrm{X})$ be a self-similar group. Then for a positive integer $n$ the action $\left(G, \mathrm{X}^{n}\right)$ is defined as the restriction of the action of the group $G$ onto the subset $\left(\mathrm{X}^{n}\right)^{*}$ of $\mathrm{X}^{*}$.

The action $\left(G, \mathbf{X}^{n}\right)$ is obviously self-similar. Let $\mathbf{X}=\{0,1, \ldots, d-1\}$ with the natural ordering. Let us order the elements of $\mathrm{X}^{n}$ lexicographically: a word $x_{1} x_{2} \ldots x_{n}$ comes before the word $y_{1} y_{2} \ldots y_{n}$ if $x_{k}<y_{k}$, where $k$ is the smallest index such that $x_{k} \neq y_{k}$. A word $x_{1} x_{2} \ldots x_{n}$ will be then on the place number $d^{n-1} x_{1}+d^{n-2} x_{2}+\cdots+x_{n}$ in the alphabet $\mathrm{X}^{n}$ (where numeration starts from 0 ).

Let $\psi_{n}: \mathbb{C} G \longrightarrow M_{d^{n} \times d^{n}}(\mathbb{C} G)$ be the matrix recursion associated with the action $\left(G, X^{n}\right)$. It follows from the definitions that the matrix $\psi_{n}(a)$ is obtained recursively from the matrix $\psi_{n-1}(a)=\left(a_{i j}\right)_{1 \leq i, j \leq d^{n-1}}$ by replacing each entry $a_{i j}$ by the $d \times d$-matrix $\psi\left(a_{i j}\right)$ (where $\psi_{1}=\psi$ ). 
EXAMPLE 9. The matrix recursion associated with the adding machine action $(\langle a\rangle, \mathrm{X})$ defined in Example 1 is given by

$$
\psi(a)=\left(\begin{array}{cc}
0 & a \\
1 & 0
\end{array}\right) .
$$

Consequently, the matrix recursion associated with the action $\left(\langle a\rangle, \mathrm{X}^{2}\right)$ is given by

$$
\psi_{2}(a)=\left(\begin{array}{cc|cc}
0 & 0 & 0 & a \\
0 & 0 & 1 & 0 \\
\hline 1 & 0 & 0 & 0 \\
0 & 1 & 0 & 0
\end{array}\right)
$$

EXAMPLE 10. The matrix recursion associated with the iterated monodromy group of $z^{2}-1$, defined in Example 2, is given by

$$
\psi(a)=\left(\begin{array}{cc}
0 & b \\
1 & 0
\end{array}\right), \quad \psi(b)=\left(\begin{array}{cc}
1 & 0 \\
0 & a
\end{array}\right),
$$

consequently as the second iteration of the matrix recursion we get the recursion

$$
\psi_{2}(a)=\left(\begin{array}{cccc}
0 & 0 & 1 & 0 \\
0 & 0 & 0 & a \\
1 & 0 & 0 & 0 \\
0 & 1 & 0 & 0
\end{array}\right), \quad \psi_{2}(b)=\left(\begin{array}{cccc}
1 & 0 & 0 & 0 \\
0 & 1 & 0 & 0 \\
0 & 0 & 0 & b \\
0 & 0 & 1 & 0
\end{array}\right) .
$$

Note that it follows directly from the definitions that if $S$ is a symmetric generating set of a self-similar group and $M=\sum_{s \in S} s$, then the matrix $\psi_{n}(s)$ is essentially the adjacency matrix of the Schreier graph $\Gamma\left(G, \mathrm{X}^{n}, S\right)$ of the action of $G$ on $\mathrm{X}^{n}$. More precisely, the adjacency matrix is obtained from the matrix $\psi_{n}(s)$ by applying the trivial representation to the elements of the matrix (i.e., replacing every group element by 1 ). In this way the matrix recursion is a systematic recurrent method of constructing the Schreier graphs and their adjacency matrices.

We hope that algebraic formalism of matrix recursions will be helpful in the study of properties of Schreier graphs and their limit fractals. Note that matrix recursions were already effectively used in computation of spectra of Schreier graphs

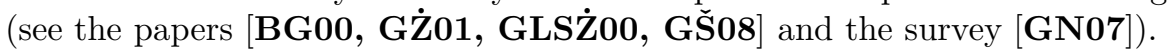

3.2. Representations of $G$ and $C\left(\mathcal{J}_{G}\right)$ on the rooted tree. Let $(G, \mathrm{X})$ be a self-similar contracting group. The action of $G$ on the set $\mathrm{X}^{*}$ induces a natural permutational unitary representation $\pi$ of $G$ on $\ell^{2}\left(\mathrm{X}^{*}\right)$. This representation is a direct sum of finite-dimensional representations $\pi_{n}$ of $G$ on $\ell^{2}\left(\mathrm{X}^{n}\right)$ for $n \geq 0$. Note also that the representation of $G$ on $\ell^{2}\left(\mathrm{X}^{n}\right)$ contains the representation of $G$ on $\ell^{2}\left(\mathrm{X}^{n-1}\right)$ as a direct summand.

Note that $\pi_{0}$ is the trivial representation mapping all elements of the group $G$ to 1 . For $n \geq 1$ and $a \in \mathbb{C} G$ the matrix of the operator $\pi_{n}(a)$ in the standard basis $\mathrm{X}^{n}$ of $\ell^{2}\left(\mathrm{X}^{n}\right)$ is equal to $\pi_{0}\left(\psi_{n}(a)\right)$, where $\psi_{n}: \mathbb{C} G \longrightarrow M_{d^{n} \times d^{n}}(\mathbb{C} G)$ is the matrix recursion associated with the action $\left(G, \mathrm{X}^{n}\right)$, i.e., the $n$th iteration of the matrix recursion $\psi: \mathbb{C} G \longrightarrow M_{d \times d}(\mathbb{C} G)$ associated with the group $(G, \mathrm{X})$.

The limit space $\mathcal{J}_{G}$ is a quotient of the space $\mathbf{X}^{-\omega}$. Consequently, the algebra of continuous complex-valued functions $C\left(\mathcal{J}_{G}\right)$ on the limit space is a sub-algebra of $C\left(\mathrm{X}^{-\omega}\right)$. Namely, $C\left(\mathcal{J}_{G}\right)$ is isomorphic to the sub-algebra of the functions which 
are constant on the asymptotic equivalence classes (see Definition 2.3). We will identify $C\left(\mathcal{J}_{G}\right)$ with this sub-algebra of $C\left(\mathrm{X}^{-\omega}\right)$.

Choose a sequence $w \in \mathbf{X}^{-\omega}$ and define a representation $\rho_{w}$ of the algebra $C\left(\mathrm{X}^{-\omega}\right) \supset C\left(\mathcal{J}_{G}\right)$ by the rule

$$
\rho_{w}(f)\left(\delta_{v}\right)=f(w v) \delta_{v},
$$

where $\delta_{v}$ is the basic vector of $\ell^{2}\left(\mathrm{X}^{*}\right)$ corresponding to the word $v \in \mathrm{X}^{*}$.

Proposition 3.1. Representation $\rho_{w}$ is bounded and faithful.

Proof. It is easy to see that $\left\|\rho_{w}(f)\right\| \leq \sup _{w \in \mathrm{X}^{-\omega}}|f(w)|=\|f\|$, hence the representation is bounded. It is faithful, since the set $\left\{w v: v \in \mathrm{X}^{*}\right\}$ is dense in $X^{-\omega}$.

We will also denote by $\rho_{w, n}$ the restriction of the representation $\rho_{w}$ onto the subspace $\ell^{2}\left(\mathbf{X}^{n}\right)$. If $w$ is fixed, then we denote $\rho_{w}$ just by $\rho$ and $\rho_{w, n}$ by $\rho_{n}$.

Suppose that $f$ is a linear combination of the characteristic functions of the cylindrical sets $\mathbf{X}^{-\omega} v$ for $v \in \mathbf{X}^{n}$. Then $\rho_{w, n}(f)$ is the diagonal matrix with the elements on the diagonal equal to the coefficients of the linear combination. For $m>n$ the matrix $\rho_{w, m}(f)$ is obtained by repeating $d^{m-n}$ times the matrix $\rho_{w, n}(f)$ along the diagonal.

We have the following description of the limit space $X^{-\omega}$ in terms of representations $\pi$ and $\rho$ (for a proof see $[\mathbf{N e k 0 8 b}]$ ).

Theorem 3.2. Let $f \in C\left(\mathrm{X}^{-\omega}\right)$. Then the following conditions are equivalent

(1) $f$ belongs to $C\left(\mathcal{J}_{G}\right)$,

(2) the operator $[\rho(f), \pi(g)]$ is compact for every $g \in G$,

(3) $\lim _{n \rightarrow \infty}\left\|\left[\rho_{n}(f), \pi_{n}(g)\right]\right\|=0$ for all $g \in G$.

One can generalize the representations $\rho_{w}$ and $\pi$ in the following way. Let $k$ be any positive number and let $W=\left\{w_{1}, \ldots, w_{k}\right\} \subset \mathbf{X}^{-\omega}$ be a set of $k$ left-infinite sequences. The corresponding representation $\rho_{W}$ of $C\left(\mathcal{J}_{G}\right)$ is just the direct sum of the representations $\rho_{w_{i}}$. Consequently, the $\rho_{W}$ is a representation on the direct sum $\ell^{2}\left(\mathbf{X}^{*}\right)^{k}$ of $k$ copies of $\ell^{2}\left(\mathbf{X}^{*}\right)$, each copy corresponding to an element $w_{i}$ of $W$. Let us denote by $\delta_{v, i}$ for $v \in \mathbf{X}^{*}$ and $i=1,2, \ldots, k$, the delta function $\delta_{v} \in \ell^{2}(v)$ in the $i$ th copy of $\ell^{2}\left(\mathrm{X}^{*}\right)$ in the direct sum $\ell^{2}\left(\mathrm{X}^{*}\right)^{k}$. The vectors $\delta_{v, i}$ form a natural basis of $\ell^{2}\left(\mathrm{X}^{*}\right)^{k}$.

We get then a natural representation $\pi_{W}$ of the algebra $M_{k \times k}(\mathbb{C} G)$ of $k \times k$ matrices over the group ring $\mathbb{C} G$. The algebra $M_{k \times k}(\mathbb{C} G)=M_{k \times k}(\mathbb{C}) \otimes \mathbb{C} G$ is the linear span of matrices $e_{i j} \otimes g$, where $e_{i j}$ is a matrix unit (with $1 \leq i, j \leq k$ ) and $g$ is an element of the group. The operators $\pi_{W}\left(e_{i j} \otimes g\right)$ acts on the space $\ell^{2}\left(\mathbf{X}^{*}\right)^{k}$ by the rule

$$
\pi_{W}\left(e_{i j} \otimes g\right)\left(\delta_{v, i}\right)=\delta_{g(v), j} .
$$

One can show that, as in Theorem 3.2, for every $a \in M_{k \times k}(\mathbb{C} G)$ and $f \in C\left(\mathcal{J}_{G}\right)$ the operator $\left[\rho_{W}(f), \pi_{W}(a)\right]$ is compact.

3.3. Quantized calculus on the limit space. Following A. Connes (see Chapter IV of [Con94]) a quantized calculus on a $*$-algebra $\mathcal{A}$ is defined by a Fredholm module in the following way. 
Definition 3.2. A Fredholm module over a $*$-algebra $\mathcal{A}$ is a representation $\rho: \mathcal{A} \longrightarrow B(H)$ of $\mathcal{A}$ by bounded operators on a Hilbert space $H$ together with an operator $F$ on $H$ such that $F^{*}=F, F^{2}=1$ and

$$
[F, \rho(a)]
$$

is compact for every $a \in \mathcal{A}$.

Fredholm module is a generalization of the notion of elliptic operator. See [Ati70, BDF73, Miš74, Kas75], where theory of such modules was developed.

Theorem 3.2 (and its analog for the representation $\rho_{W}$ ) shows that we get a natural family of Fredholm modules over $\mathcal{A}=C\left(\mathcal{J}_{G}\right)$.

For example, we may always take the involution $S=\left(\begin{array}{ll}0 & 1 \\ 1 & 0\end{array}\right) \in M_{2 \times 2}(\mathbb{C}) \subset$ $M_{2 \times 2}(\mathbb{C} G)$ and consider a representations $\rho=\rho_{\left\{w_{1}, w_{2}\right\}}$ and $\pi=\pi_{\left\{w_{1}, w_{2}\right\}}$ (where $w_{1}$ and $w_{2} \in \mathbf{X}^{-\omega}$ represent different points of $\left.\mathcal{J}_{G}\right)$. Then the pair $(\rho, \pi(S))$ is a Fredholm module. In some cases other choices of $F$ are natural, for instance $\rho(g)$, where $g \in G$ is a non-trivial involution.

The following is an analog of similar properties of the quantized calculus on the circle described in [Con94] (see Proposition IV.3.14).

THEOREM 3.3. Let $f$ be a hyperbolic rational function such that the Julia set of $f$ is a subset of $\mathbb{C}$ (i.e., does not contain $\infty)$. Let $(G, \mathrm{X})$ be the iterated monodromy group of $f$ together with a standard self-similar action on $\mathrm{X}^{*}$. Let $Z: \mathcal{J}_{G} \longrightarrow \mathbb{C}$ be the associated canonical homeomorphism of $\mathcal{J}_{G}$ with the Julia set of $f$, seen as a continuous function on $\mathcal{J}_{G}$.

Let $\rho, \pi$ and $S$ be either the representations $\rho_{w}$ of $C\left(\mathcal{J}_{G}\right), \pi$ of $G$ and a non-trivial element $S \in G$, or the representations $\rho_{\left\{w_{1}, w_{2}\right\}}$ of $C\left(\mathcal{J}_{G}\right), \pi_{\left\{w_{1}, w_{2}\right\}}$ of $M_{2 \times 2}(\mathbb{C} G)$ and $S=\left(\begin{array}{ll}0 & 1 \\ 1 & 0\end{array}\right)$, where $w_{1}, w_{2} \in \mathrm{X}^{-\omega}$ represent different points of $\mathcal{J}_{G}$. Then the number

$$
\inf \left\{p>1:[\rho(Z), \pi(S)] \in \mathcal{L}_{p}\right\}
$$

is equal to the Hausdorff dimension of the Julia set of $f$, where $\mathcal{L}_{p}$ is the Schatten ideal $\left\{A \in B(H): \operatorname{Tr}\left(|A|^{p}\right)<\infty\right\}$.

A proof of this theorem (which is based on a result of C. T. McMullen [McM00]) will be given in a separate publication.

3.4. Constant sequences. If we take a constant sequence $w=\ldots x_{0} x_{0} x_{0}$, then $w x_{0}=w$ and hence the set $w \mathbf{X}^{n-1}=\left\{w v: v \in \mathbf{X}^{n-1}\right\}$ is contained in $w \mathbf{X}^{n}$. Then restriction $\rho_{n}$ of the representation $\rho$ onto $\ell^{2}\left(\mathrm{X}^{n}\right)$ will contain the restriction $\rho_{n-1}$ of $\rho$ onto $\ell^{2}\left(\mathrm{X}^{n-1}\right)$. As a direct limit of the representations $\rho_{n}$, we get a faithful bounded representation $\rho_{*}$ of the algebra $C\left(\mathcal{J}_{G}\right)$ on the space

$$
\ell^{2}\left(\bigcup_{n \geq 0} w \mathrm{X}^{n}\right)=\ell^{2}\left(w \mathrm{X}^{*}\right) .
$$

Let us denote $V_{n}=V_{n}(w)=w \mathrm{X}^{n}$ and $V_{*}=V_{*}(w)=\bigcup_{n \geq 0} V_{n}$.

The identifications of $\ell^{2}\left(X^{n-1}\right)$ with a subspace of $\ell^{2}\left(X^{n}\right)$ coming from the inclusion $w \mathrm{X}^{n-1} \subset w \mathrm{X}^{n}$ is the linear embedding induced by the map

$$
v \mapsto x_{0} v
$$


These identifications do not agree with the representation $\pi$ of $G$, so we will not get any direct limit representation of $G$ on $\ell^{2}\left(w \mathrm{X}^{*}\right)$.

More generally, suppose that we have a set $W=\left\{w_{1}, w_{2}, \ldots, w_{k}\right\} \subset \mathbf{X}^{-\omega}$ of sequences, letters $x_{1}, x_{2}, \ldots, x_{k}$ and indices $1 \leq n_{1}, n_{2}, \ldots, n_{k} \leq k$ such that

$$
w_{i}=w_{n_{i}} x_{i}
$$

for all $1 \leq m \leq k$. Consider the associated representations $\rho_{W}$ of $C\left(\mathcal{J}_{G}\right)$ and $\pi$ of $M_{k \times k}(\mathbb{C} G)$.

We will also denote by $\rho_{n}$ the restriction of $\rho$ onto the direct sum of the spaces $\ell^{2}\left(w_{i} \mathrm{X}^{n}\right)$ for $i=1, \ldots, k$. Since $w_{n_{i}} x_{i}=w_{i}$, we have $w_{i} \mathrm{X}^{n} \subset w_{n_{i}} \mathrm{X}^{n+1}$, hence the space $\ell^{2}\left(w_{i} \mathrm{X}^{n}\right)$ is a subspace of $\ell^{2}\left(w_{n_{i}} \mathrm{X}^{n+1}\right)$ in a natural way. Hence we also get a natural identification of $\rho_{n}$ with a sub-representation of $\rho_{n+1}$ and the inductive limit representation $\rho_{*}$ of $C\left(\mathcal{J}_{G}\right)$ on $\ell^{2}\left(W \mathrm{X}^{*}\right)$.

Denote by $V_{n}$ the set of points of $\mathcal{J}_{G}$ represented by the sequences of the form $w_{i} v$ for $v \in \mathbf{X}^{n}$. Note that $V_{n}$ may have less points than $k\left|\mathbf{X}^{n}\right|$, since different sequences may represent the same points. We also have $V_{n} \subset V_{n+1}$ and hence can define the direct limit set $V_{*}=\bigcup_{n>0} V_{n}$. The space $\ell^{2}\left(V_{n}\right)$ is naturally isomorphic to the $\rho_{W}$-invariant subspace of $\ell^{2}\left(W X^{n}\right)$ consisting of functions assuming equal values on sequences representing equal points of $\mathcal{J}_{G}$. Similarly, the space $\ell^{2}\left(V_{*}\right)$ is naturally a subspace of $\ell^{2}\left(W X^{*}\right)$.

In some sense the inductive limit representations $\rho_{*}$ of $C\left(\mathcal{J}_{G}\right)$ on $\ell^{2}\left(W \mathrm{X}^{*}\right)$ or $\ell^{2}\left(V_{*}\right)$ are more natural than the direct sums $\rho_{W}$ of finite dimensional representations $\rho_{n}$. However, there is no natural representation of $G$ on these spaces. Our idea of constructing analysis on fractals is to use the representations $\pi_{n}$ of $M_{k \times k}(\mathbb{C} G)$ in order to pass to some limit object which agrees with the representation $\rho_{*}$. We will see in the subsequent sections of our paper how this idea works in constructions of Dirichlet forms and Laplacians on some limit spaces of self-similar groups.

\section{Laplacians, Dirichlet forms and resistance forms}

4.1. Dirichlet forms. A nonnegative real-valued symmetric quadratic form $\mathcal{E}$ with a domain $\operatorname{Dom}(\mathcal{E}) \subset L^{2}(\mathcal{X}, \nu)$ is called a Dirichlet form if the following conditions are satisfied:

(1) $\operatorname{Dom}(\mathcal{E})$ is dense in $L^{2}(\mathcal{X}, \nu)$ in the norm $\|\cdot\|$ of $L^{2}(\mathcal{X}, \nu)$;

(2) $\operatorname{Dom}(\mathcal{E})$ is a complete Hilbert space in the norm $\sqrt{\mathcal{E}+\|\cdot\|^{2}}$;

(3) [Markov property] for any $u \in \operatorname{Dom}(\mathcal{E}), v=\min \{\max \{0, u\}, 1\} \in \operatorname{Dom}(\mathcal{E})$ and $\mathcal{E}(v, v) \leqslant \mathcal{E}(u, u)$

(4) $\mathcal{X}$ is a locally compact separable metric space and $\nu$ is a positive Radon measure such that $\operatorname{supp}[\nu]=\mathcal{X}$;

A Dirichlet form is called regular if there is a subset $\mathcal{C} \subset \operatorname{Dom}(\mathcal{E}) \cap C_{0}(\mathcal{X})$ such that $\mathcal{C}$ is dense in $\operatorname{Dom}(\mathcal{E})$ in the norm $\sqrt{\mathcal{E}+\|\cdot\|^{2}}$ and $\mathcal{C}$ is dense in $C_{0}(\mathcal{X})$ in the uniform norm.

A Dirichlet form is called local if $\mathcal{E}(u, v)=0$ for any $u, v \in \operatorname{Dom}(\mathcal{E})$ such that $\operatorname{supp}[u]$ and $\operatorname{supp}[v]$ are disjoint compact sets.

The standard references on Dirichlet forms are books [BH91, FŌT94].

4.2. Resistance forms. Below we restate the definition of a resistance form in [Kig03] (see also [Kig01]). 
A pair $(\mathcal{E}, \operatorname{Dom}(\mathcal{E}))$ is called a resistance form on a countable set $V_{*}$ if it satisfies the following conditions.

$(\mathrm{RF} 1) \operatorname{Dom}(\mathcal{E})$ is a linear subspace of $\ell\left(V_{*}\right)$ containing constants, $\mathcal{E}$ is a nonnegative symmetric quadratic form on $\operatorname{Dom}(\mathcal{E})$, and $\mathcal{E}(u, u)=0$ if and only if $u$ is constant on $V_{*}$.

(RF2) Let $\sim$ be the equivalence relation on $\operatorname{Dom}(\mathcal{E})$ defined by $u \sim v$ if and only if $u-v$ is constant on $V_{*}$. Then $(\mathcal{E}, \operatorname{Dom}(\mathcal{E}) / \sim)$ is a Hilbert space.

(RF3) Markov property (see Subsection 4.1).

(RF4) For any finite subset $V \subset V_{*}$ and for any $v \in \ell(V)$ there exists $u \in \operatorname{Dom}(\mathcal{E})$ such that $\left.u\right|_{V}=v$.

(RF5) For any $p, q \in V_{*}$

$$
\sup \left\{\frac{(u(p)-u(q))^{2}}{\mathcal{E}(u, u)}: u \in \operatorname{Dom}(\mathcal{E}), \mathcal{E}(u, u)>0\right\}<\infty .
$$

This supremum is denoted by $R(p, q)$ and called the effective resistance between $p$ and $q$.

For any finite subset $U \subset V_{*}$ the finite dimensional Dirichlet form $\mathcal{E}_{U}$ on $U$ is defined by

$$
\mathcal{E}_{U}(f, f)=\inf \left\{\mathcal{E}(g, g): g \in \operatorname{Dom}(\mathcal{E}),\left.g\right|_{U}=f\right\},
$$

which exists (see, for instance, [Kig03]), and moreover there is a unique $g$ for which the inf is attained. The Dirichlet form $\mathcal{E}_{U}$ is called the trace of $\mathcal{E}$ on $U$, and denoted

$$
\mathcal{E}_{U}=\operatorname{Trace}_{U}(\mathcal{E}) .
$$

By the definition, if $U_{0} \subset U_{1}$ then $\mathcal{E}_{U_{0}}$ is the trace of $\mathcal{E}_{U_{1}}$ on $U_{0}$, that is

$$
\mathcal{E}_{U_{0}}=\operatorname{Trace}_{U_{0}}\left(\mathcal{E}_{U_{1}}\right) \text {. }
$$

Note that if the matrix of $\mathcal{E}_{U_{1}}$ is denoted by $D_{1}$ and is written in the block form

$$
D_{1}=\left(\begin{array}{cc}
A & B \\
C & D
\end{array}\right) \text {, }
$$

according to the decomposition $U_{1}=U_{0} \cup\left(U_{1} \backslash U_{0}\right)$, then the trace of $\mathcal{E}_{U_{1}}$ on $U_{0}$ is given by the matrix

$$
D_{0}=A-B D^{-1} C .
$$

This is the formula for the well known Schur complement. In this setting the Schur complement appears because the minimization problem in the definition of the trace (4.1), by elementary analysis and linear algebra, leads to

$$
C\left(\left.g\right|_{U_{0}}\right)+D\left(\left.g\right|_{U_{1} \backslash U_{0}}\right)=0
$$

which implies

$$
\left.g\right|_{U_{1} \backslash U_{0}}=-D^{-1} C\left(\left.g\right|_{U_{0}}\right)=-D^{-1} C f .
$$

For some relations of Schur complements to theory of self-similar groups, see [GN07].

THEOREM 4.1 (Kigami [Kig03]). Suppose that $V_{n}$ are finite subsets of $V_{*}$ and that $\bigcup_{n=0}^{\infty} V_{n}$ is $R$-dense in $V_{*}$. Then

$$
\mathcal{E}(f, f)=\lim _{n \rightarrow \infty} \mathcal{E}_{V_{n}}(f, f)
$$


for any $f \in \operatorname{Dom}(\mathcal{E})$, where the limit is actually non-decreasing. Is particular, $\mathcal{E}$ is uniquely defined by the sequence of its finite dimensional traces $\mathcal{E}_{V_{n}}$ on $V_{n}$.

TheOREM 4.2 (Kigami [Kig03]). Suppose that $V_{n}$ are finite sets, for each $n$ there is a resistance form $\mathcal{E}_{V_{n}}$ on $V_{n}$, and this sequence of finite dimensional forms is compatible in the sense that each $\mathcal{E}_{V_{n}}$ is the trace of $\mathcal{E}_{V_{n+1}}$ on $V_{n}$, where $n=0,1,2, \ldots$. Then there exists a resistance form $\mathcal{E}$ on $V_{*}=\bigcup_{n=0}^{\infty} V_{n}$ such that

$$
\mathcal{E}(f, f)=\lim _{n \rightarrow \infty} \mathcal{E}_{V_{n}}(f, f)
$$

for any $f \in \operatorname{Dom}(\mathcal{E})$, and the limit is actually non-decreasing.

Note that the effective resistance $R$ is a metric on $V_{*}$, and that any function in $\operatorname{Dom}(\mathcal{E})$ is $R$-continuous. Let $\Omega$ be the $R$-completion of $V_{*}$. Then any $u \in \operatorname{Dom}(\mathcal{E})$ has a unique $R$-continuous extension to $\Omega$. We will somewhat abuse notation by speaking about the resistance form $\mathcal{E}$ defined on $\Omega$, and consider $\operatorname{Dom}(\mathcal{E}) \subset C_{R}(\Omega)$, where $C_{R}(\Omega)$ is the space of $R$-continuous functions.

Now assume that $V_{*}$ is a subset of some metric space $\mathcal{X}$, and that $V_{*}$ is dense in $\mathcal{X}$. Since it is not necessarily true that the metric of $\mathcal{X}$ on $V_{*}$ is equivalent to the $R$-metric, the spaces $\Omega$ and $\mathcal{X}$ may have little relation, and in particular, may not be embedded one into the other. In some situations it is very useful to distinguish these two spaces, for instance, when $\mathcal{X}$ is compact, and $\Omega$ is not. However, in what follows we assume that the induced topology of $\mathcal{X}$ on $V_{*}$ coincides with the $R$ topology on $V_{*}$, and so we can identify $\Omega$ and $\mathcal{X}$. Moreover, we assume that $\Omega=\mathcal{X}$ is compact. In such a situation the resistance form $\mathcal{E}$ is called regular. Note that the notion of regularity of a resistance form is distinct from a notion of regularity of a Dirichlet form.

Theorem 4.3 (Kigami [Kig03]). A regular resistance form $\mathcal{E}$ on a compact metric space $\mathcal{X}$ is a regular Dirichlet form on $L^{2}(\mathcal{X}, \nu)$ for any finite positive Radon measure $\nu$ such that $\operatorname{supp}[\nu]=\mathcal{X}$.

It can be seen immediately from the definitions that not every Dirichlet form is a resistance form, since there are Dirichlet forms such that all finite sets have zero capacity. However, on a finite set a resistance form is the same as a conservative Dirichlet form. Note that any resistance form on a finite set $U$ can be written uniquely as

$$
\mathcal{E}_{U}(f, f)=\sum_{x, y \in U} C_{x, y}(f(x)-f(y))^{2},
$$

where $C_{x, y} \geqslant 0$ are often called conductances between points $x$ and $y$.

4.3. Dirichlet resistance forms on p.c.f. self-similar fractals. The following definition is from [Kig93, Kig01].

Definition 4.1. We say that a compact connected metrizable space $\mathcal{X}$ is a self-similar set if there is a continuous surjection

$$
\Pi: X^{\omega} \rightarrow \mathcal{X}
$$

where $\mathbf{X}=\{0,1, \ldots, d-1\}$, and continuous injective maps

$$
\psi_{0}, \ldots, \psi_{d-1}: \mathcal{X} \rightarrow \mathcal{X}
$$

such that

$$
\psi_{i}\left(\Pi\left(x_{1} x_{2} \ldots\right)\right)=\Pi\left(i x_{1} x_{2} \ldots\right)
$$


for all $i \in \mathrm{X}$. Moreover, we say that $\mathcal{X}$ is a post critically finite (p.c.f.) self-similar set if the post critical set

$$
\mathcal{P}=\bigcup_{n \geqslant 1} \mathrm{~s}^{n}\left(\bigcup_{i, j \in \mathrm{X}, i \neq j} \Pi^{-1}\left(\psi_{i}(\mathcal{X}) \cap \psi_{j}(\mathcal{X})\right)\right)
$$

is finite.

We denote $V_{0}=\Pi(\mathcal{P}), V_{w}=\psi_{w}\left(V_{0}\right), V_{n}=\bigcup_{w \in \mathrm{X}^{n}} V_{w}$, and

$$
V_{*}=\bigcup_{n \geqslant 0} V_{n}
$$

Here for a finite word $w=w_{1} \ldots w_{n} \in \mathbf{X}^{n}$ we denote

$$
\psi_{w}=\psi_{w_{1}} \circ \cdots \circ \psi_{w_{n}} .
$$

Note that, by definition, each $\psi_{i}$ maps $V_{*}$ into itself injectively.

The sets $\mathcal{X}_{w}=\psi_{w}(\mathcal{X})$ are called cells, and the set $V_{0}$ can be called the vertex boundary of $\mathcal{X}$. In terms of analysis, $V_{0}$ is the set where boundary conditions for various partial differential equations on $\mathcal{X}$ are usually imposed (see [Str06]).

Note that in many references, such as [Hve05], the term cell has the same meaning as the term tile in most of our paper. Therefore, we can use terms cells and tiles interchangeably.

4.4. Strictly p.c.f. groups. We see that the notion of a finitely ramified set (see Subsection 6.1) and of a p.c.f. self-similar set is very similar to the notion of selfsimilar groups of bounded automorphisms (more precisely, to their limit spaces). The sets $\mathcal{X}_{w}$ are analogs of the tiles $\mathcal{T}_{w}$. We also have that the intersection $\mathcal{T}_{w} \cap \mathcal{T}_{w^{\prime}}$ is finite, that $\mathcal{J}_{G}=\bigcup_{w \in \mathrm{X}^{n}} \mathcal{T}_{w}$; and we also have the shift map s : $\mathcal{T}_{w x} \longrightarrow \mathcal{T}_{w}$, which can be identified with $\psi_{x}^{-1}$. The only difference is that we do not have a metric structure on the limit space $\mathcal{J}_{G}$ and, perhaps more importantly, the maps $\mathrm{s}: \mathcal{T}_{w x} \longrightarrow \mathcal{T}_{w}$ may be non-injective (that is, $\mathcal{J}_{G}$ may not be self-homeomorphic in the terminology of [Hve05], see Subsection 6.1). One can show, however, that the map $\mathrm{s}$ is injective on the interior of the tile $\mathcal{T}_{w x}$.

We adopt, therefore, the following definition.

Definition 4.2. We say that a self-similar group $(G, \mathrm{X})$ is strictly p.c.f. if it is a subgroup of the group of bounded automorphisms $\mathcal{B}(\mathrm{X})$ and every element of the nucleus of $G$ changes at most one letter in every word $v \in \mathrm{X}^{*}$.

Proposition 4.4. If $(G, \mathrm{X})$ is strictly p.c.f., then restriction of the shift $\mathrm{s}$ : $\mathcal{J}_{G} \longrightarrow \mathcal{J}_{G}$ onto the tile $\mathcal{T}_{v x}$ is a homeomorphism $\mathrm{s}: \mathcal{T}_{v x} \longrightarrow \mathcal{T}_{v}$ for every $v \in \mathrm{X}^{*}$ and $x \in \mathrm{X}$. In particular, the tiles are homeomorphic to the limit space $\mathcal{J}_{G}$.

Proof. It is sufficient to show that for every $v \in \mathrm{X}^{*}$ the map $\mathrm{s}^{|v|}: \mathcal{T}_{v} \longrightarrow$ $\mathcal{J}_{G}$ is bijective. If it is not, then there exist non-equivalent sequences $\ldots x_{2} x_{1} v$ and $\ldots y_{2} y_{1} v$, such that $\ldots x_{2} x_{1}$ and $\ldots y_{2} y_{1}$ are equivalent. Then there exists a sequence $g_{k}$ of the elements of the nucleus such that $g_{k}\left(x_{k} \ldots x_{1}\right)=y_{k} \ldots y_{1}$. Since $\ldots x_{2} x_{1}$ and $\ldots y_{2} y_{1}$ are different, $g_{k}$ changes at least one letter in $x_{k} \ldots x_{1}$ for all sufficiently big $k$. But it is not allowed to change more than one letter in a word, hence $g_{k}\left(x_{k} \ldots x_{1} v\right)=y_{k} \ldots y_{1} v$ for all sufficiently big $k$, which implies that $\ldots x_{2} x_{1} v$ and $\ldots y_{2} y_{1} v$ are equivalent. 
4.5. Self-similar forms. In this subsection $\mathcal{X}$ is a p.c.f. self-similar set, and $V_{*}$ as defined above. A resistance form $\mathcal{E}$ on $V_{*}$ is self-similar with energy renormalization factors $\rho=\left(\rho_{0}, \ldots, \rho_{d-1}\right)$ if for any $f \in \operatorname{Dom}(\mathcal{E})$ we have

$$
\mathcal{E}(f, f)=\sum_{i=0}^{d-1} \rho_{i} \mathcal{E}\left(f_{i}, f_{i}\right)
$$

Here we use the notation $f_{w}=f \circ \psi_{w}$ for any $w \in W_{*}$.

The energy renormalization factors, or weights, $\rho=\left(\rho_{0}, \ldots, \rho_{d-1}\right)$ are often also called conductance scaling factors because of the relation of resistance forms and electrical networks. They are reciprocals of the resistance scaling factors $r_{j}=\frac{1}{\rho_{j}}$.

For a set of energy renormalization factors $\rho=\left(\rho_{0}, \ldots, \rho_{d-1}\right)$ and any resistance form $\mathcal{E}_{0}$ on $V_{0}$ define the resistance form $\Psi_{\rho}\left(\mathcal{E}_{0}\right)$ on $V_{1}$ by

$$
\Psi_{\rho}\left(\mathcal{E}_{0}\right)(f, f)=\sum_{i=0}^{d-1} \rho_{i} \mathcal{E}_{0}\left(g_{i}, g_{i}\right),
$$

where

$$
g_{i}=\left.f\right|_{\psi_{i}\left(V_{0}\right)} \circ \psi_{i}^{-1} .
$$

Then $\Lambda\left(\mathcal{E}_{0}\right)$ is defined as the trace of $\Psi_{\rho}\left(\mathcal{E}_{0}\right)$ on $V_{0}$ :

$$
\Lambda\left(\mathcal{E}_{0}\right)=\operatorname{Trace}_{V_{0}} \Psi_{\rho}\left(\mathcal{E}_{0}\right) .
$$

The next two propositions were originally obtained in [Kig93, Kus89, Lin91], and their proof can also be found in [Bar98, Kig01], for the p.c.f. self-similar case. They can be applied to the self-similar finitely ramified case because of [Kig03, Tep08].

Proposition 4.5. If $\mathcal{E}$ is self-similar then $\mathcal{E}_{0}=\Lambda\left(\mathcal{E}_{0}\right)$.

Proposition 4.6. If $\mathcal{E}_{0}$ is such that

$$
\mathcal{E}_{0}=\Lambda\left(\mathcal{E}_{0}\right)
$$

then there is a self-similar resistance form $\mathcal{E}$ such that $\mathcal{E}_{0}$ is the trace of $\mathcal{E}$ on $V_{0}$.

Note that these propositions reduce the question of existence of a self-similar Dirichlet form, which is an infinite dimensional problem, to the finding of a solution to the nonlinear eigenvector equation for the map $\Lambda$ (see the above reference and also [Met03, Sab97]).

Proposition 4.7. If $\rho_{i}>1$ for all $i$ then $\mathcal{E}$ is regular, and in particular $\Omega=\mathcal{X}$ is compact.

Proof. If $\operatorname{diam}_{R}(\cdot)$ denotes the diameter of a set in the effective resistance metric $R$, and $\rho_{w}=\rho_{w_{1}} \ldots \rho_{w_{n}}$ for any finite word $w=w_{1} \ldots w_{n} \in W_{n}$, then

$$
\operatorname{diam}_{R}(\mathcal{X}) \geqslant \rho_{w} \operatorname{diam}_{R}\left(\mathcal{X}_{w}\right)
$$

by the self-similarity of the resistance form and the definition of the metric $R$. 
4.6. Symmetries of the fractal. A group $\mathfrak{G}$ is said to act on a finitely ramified fractal $\mathcal{X}$ if each $g \in \mathfrak{G}$ is a homeomorphism of $\mathcal{X}$ such that $g\left(V_{n}\right)=V_{n}$ for all $n \geqslant 0$.

Proposition 4.8. If a group $\mathfrak{G}$ acts on a finitely ramified fractal $\mathcal{X}$ then for each $g \in \mathfrak{G}$ and each $n$-cell $\mathcal{X}_{\alpha}$ and $g\left(\mathcal{X}_{\alpha}\right)$ is an $n$-cell.

REMARK. Note that the group $\mathfrak{G}$ has no direct relation with self-similar groups. The self-similar group $G$ does not act on its limit space $\mathcal{J}_{G}$. The group $G$ should be thought of as a kind of "infinitesimal action" on the limit space $\mathcal{J}_{G}$ (see Theorem 3.2), while the group $\mathfrak{G}$ is an automorphism group of $\mathcal{J}_{G}$. For instance, in the case of the Sierpiński gasket the group $\mathfrak{G} \cong S_{3}$ is isomorphic to the symmetric permutation group of three elements.

Proposition 4.9. Suppose a group $\mathfrak{G}$ acts on a self-similar finitely ramified fractal $\mathcal{X}$, and its action on $V_{0}$ is doubly transitive. Then there exists a unique, up to a constant, $\mathfrak{G}$-invariant self-similar resistance form $\mathcal{E}$ with equal energy renormalization weights and

$$
\mathcal{E}_{0}(f, f)=\sum_{x, y \in V_{0}}(f(x)-f(y))^{2} .
$$

It is easy to see that, up to a constant, $\mathcal{E}_{0}$ is the only $\mathfrak{G}$-invariant resistance form on $V_{0}$. Let $\rho=(1, \ldots, 1)$. Then $\Lambda\left(\mathcal{E}_{0}\right)$ is also $\mathfrak{G}$-invariant and so $\operatorname{Trace}_{V_{0}} \Psi_{\rho}\left(\mathcal{E}_{0}\right)=$ $c \mathcal{E}_{0}$ for some $c$.

4.7. Existence and uniqueness of self-similar Dirichlet forms and properties of the $\Lambda$ map. In this subsection we assume that the energy renormalization factors $\rho=\left(\rho_{0}, \ldots, \rho_{d-1}\right)$ are fixed.

Let $\mathbb{D}$ be the cone of Dirichlet forms on $V_{0}$. It is easy to see that $\Lambda$ maps $\mathbb{D}$ into itself, and we are interested in the existence and uniqueness of its (nonlinear) eigenvectors. The cone $\mathbb{D}$ spans the real vector space $\mathbb{B}=\mathbb{D}-\mathbb{D}$ which we endow with the norm $\left\|\mathcal{E}_{0}\right\|_{\mathbb{B}}=\sup \left\{\left|\mathcal{E}_{0}(f)\right| ;\|f\|=1\right\}$. Set $\mathbb{P}=\{\mathcal{E} \in \mathbb{B} \mid \mathcal{E}(\cdot) \geq 0\}$ which is the cone of positive semidefinite forms. The cone $\mathbb{P}$ defines a partial ordering $\mathcal{E} \leq \mathcal{F}$ on $\mathbb{B}$ by $\mathcal{F}-\mathcal{E} \in \mathbb{P}$. It is the pointwise ordering of positive semidefinite quadratic forms. Then $\Lambda$ has the following properties.

(1) $\Lambda: \mathbb{P} \rightarrow \mathbb{P}, \mathbb{P}^{\circ} \rightarrow \mathbb{P}^{\circ}$.

(2) $\Lambda(\alpha \mathcal{E})=\alpha \Lambda(\mathcal{E})$ and $\Lambda(\mathcal{E}+\mathcal{F}) \geq \Lambda(\mathcal{E})+\Lambda(\mathcal{F})$ for all $\mathcal{E}, \mathcal{F} \in \mathbb{P}$ and $\alpha \geq 0$.

(3) $\Lambda$ is continuous on $\mathbb{D} \cup \mathbb{P}^{\circ}$.

For $\mathcal{E}, \mathcal{F} \in \mathbb{P}^{\circ}$ there exists the biggest lower bound of $\mathcal{E} / \mathcal{F}$,

$$
m(\mathcal{E} / \mathcal{F})=\sup \{\alpha>0 \mid \alpha \mathcal{F} \leq \mathcal{E}\} \in(0,+\infty) .
$$

and the smallest upper bound $M(\mathcal{E} / \mathcal{F})$ is equal to $m(\mathcal{F} / \mathcal{E})^{-1}$. On $\mathbb{P}^{\circ}$ we define Hilbert's projective metric $h$ by

$$
h(\mathcal{E}, \mathcal{F})=\ln \frac{M(\mathcal{E} / \mathcal{F})}{m(\mathcal{E} / \mathcal{F})} .
$$

It is a pseudo distance on $\mathbb{P}^{\circ}$. Moreover,

(1) $h(\alpha \mathcal{E}, \beta \mathcal{F})=h(\mathcal{E}, \mathcal{F})$ for all $\alpha, \beta>0$, and $h(\mathcal{E}, \mathcal{F})=0$ if and only if there exists $\alpha>0$ such that $\mathcal{E}=\alpha \mathcal{F}$.

(2) For every $\mathcal{E}, \mathcal{F} \in \mathbb{P}^{\circ}$ the distance $h(\mathcal{E}, \mathcal{F})$ tends to $\infty$ when $\mathcal{F}$ tends to $\partial \mathbb{P}$. 
Following the methods developed in [Met03, Met04, Sab97, HMT06], one can show that if there are two distinct eigenvectors, then there is an $h$-geodesic path of eigenvectors connecting them, and this geodesic can be extended up to the boundary. Thus, one has existence and uniqueness if the the boundary $\partial \mathbb{D}$ is repulsive, and therefore there are no eigenvectors near the boundary. A unique irreducible $\Lambda$-eigenvectors is the unique zero of a functional $q: \mathbb{H} \rightarrow \mathbb{R}_{+}$, derived from a hyperbolic distance $h$ on a projective space $\mathbb{H}=\mathbb{P}^{\circ} / \mathbb{R}_{+}$,

$$
q(\mathcal{E})=h(\Lambda(\mathcal{E}), \mathcal{E})
$$

One has the following facts.

(1) $\Lambda$ is $h$-non expansive on $\mathbb{P}^{\circ}$, that is, lower $q$-level sets are $\Lambda$-invariant.

(2) $\Lambda$ has a unique eigenvector $\mathcal{F} \in \mathbb{H}$ if and only if $\left.q\right|_{\mathbb{H}}$ vanishes only at $\mathcal{F}$.

(3) When a $\Lambda$-forward orbit started in $\mathbb{H}$ is contained in $B_{r}(\mathcal{E})$ for some $r>0$ and $\mathcal{E} \in \mathbb{H}$, then there exists a $\Lambda$-eigenvector in $B_{3 r}(\mathcal{E}) \cap \mathbb{H}$. Here $B_{r}(\mathcal{E})$ is the closed $h$-ball of radius $r>0$ centered at $\mathcal{E} \in \mathbb{P}^{\circ}$.

One can show that $\Lambda$ has more than one eigenvector in $\mathbb{H}$ if and only if $q$ vanishes on a connected set which accumulates at $\partial \mathbb{P}$.

All of the above properties are invariant under the action of the symmetry group $\mathfrak{G}$ (see Subsection 4.6). Therefore, we have the following proposition.

Proposition 4.10. If there are no eigenvectors of $\Lambda$ on the boundary $\partial \mathbb{D}_{\mathfrak{G}}$ of the cone $\mathbb{D}_{\mathfrak{G}}$ of $\mathfrak{G}$-invariant Dirichlet forms, then there is a unique eigenvector in the interior of $\mathbb{D}_{\mathfrak{G}}$.

In the case of a finitely ramified self-similar set, by Propositions 4.5 and 4.6, one can obtain all the self-similar Dirichlet forms by solving the finite dimensional nonlinear eigenvalue equation $\mathcal{E}_{0}=\Lambda\left(\mathcal{E}_{0}\right)$. In the case of complete symmetry, the Proposition 4.9 gives an easy answer to our Questions 1 and 2. In our example, this can be immediately applied to the Sierpiński gasket. It also can be applied to the case of an interval considered as a self-similar set, which is of course closely related to the example of the circle. If Proposition 4.9 is not applicable, then the situation become more difficult. The general existence and uniqueness results are known for nested fractals ([Lin91, Bar98, Kig01], for self-similar p.c.f. fractals with three boundary points (see [Pei06]) and in some other situations, such is in Theorem 6.1 (see [HMT06]) and in [Pei08]. In examples below we show how the methods of [Met03, Sab97] can be applied to the pillow fractal (see Figure 7). In general, one can not expect $\mathcal{J}_{G}$ to be finitely ramified self-similar even in the case of groups generated by bounded automata, but there are useful graph-directed constructions (see [HMT06, Met04]), which we illustrate in the Basilica example.

\section{Examples of Laplacians on limit spaces}

5.1. The circle. We begin not with the circle, but with the case of the unit interval $[0,1]$. In this case we can define contractions $\psi_{0}(x)=\frac{1}{2} x$ and $\psi_{1}(x)=$ $\frac{1}{2} x+\frac{1}{2}$ and see that

$$
[0,1]=\psi_{0}([0,1]) \cup \psi_{1}([0,1]),
$$

which give us a p.c.f. self-similar structure for $[0,1]$. In particular, $V_{0}=\{0,1\}$ and $V_{1}=\left\{0, \frac{1}{2}, 1\right\}$. Using the approach of Subsection 4.3 and the Schur complement 
(4.2), the formula (4.4) reduces to the matrix equation

$$
\frac{1}{\rho}\left(\begin{array}{rr}
1 & -1 \\
-1 & 1
\end{array}\right)=\left(\begin{array}{ll}
1 & 0 \\
0 & 1
\end{array}\right)-\left(\begin{array}{l}
-1 \\
-1
\end{array}\right)(2)^{-1}\left(\begin{array}{ll}
-1 & -1
\end{array}\right)
$$

which implies $\rho=2$. Note that this is a particular case of Proposition 4.9.

This almost trivial computation can be placed into the context of self-similar groups via the example of the binary adding machine, see Examples 1 and 9. As we have already seen above, the circle $\mathbb{R} / \mathbb{Z}$, which is the same as the interval $[0,1]$ with identified endpoints, is the limit space of the binary adding machine action of the infinite cyclic group, which is given by the matrix recursion

$$
\psi(a)=\left(\begin{array}{cc}
0 & a \\
1 & 0
\end{array}\right) .
$$

Consider the sequence $w=\ldots 000=0^{-\omega} \in \mathbf{X}^{-\omega}$ and let $\rho=\rho_{w}$ and $\pi$ be the corresponding representations of $C\left(\mathcal{J}_{G}\right)$ and $G=\langle a\rangle$ on $\ell^{2}\left(\mathrm{X}^{*}\right)$, as in 3.2. We denote by $\rho_{n}$ and $\pi_{n}$ the restrictions of the representations on the subspaces $\ell^{2}\left(\mathrm{X}^{n}\right)$.

Recall that $\rho_{n}$ becomes a sub-representation of $\rho_{n+1}$, if we identify $\ell^{2}\left(\mathrm{X}^{n}\right)$ with the subspace of $\ell^{2}\left(\mathrm{X}^{n+1}\right)$ by the map

$$
L_{0}: v \mapsto 0 v: \mathrm{X}^{n} \longrightarrow \mathrm{X}^{n+1} .
$$

Denote by $V_{n}$ the image of $\mathrm{X}^{n} w$ in $\mathcal{J}_{G}$. It is equal to the set $\left\{k / 2^{n}: k=\right.$ $\left.0,1, \ldots 2^{n}-1\right\} \subset \mathbb{R} / \mathbb{Z}$. Then $L_{0}$ induces the identical embedding of $V_{n}$ into $V_{n+1} \supset$ $V_{n}$ and the matrix $\rho_{n}(f)$ is the diagonal matrix with the entries on the diagonal equal to the values of $f$ on the corresponding points of $V_{n}$.

Consider the forms $\mathcal{E}_{n}(f, f)$ on $\ell^{2}\left(\mathrm{X}^{n}\right)$ given by the matrices

$$
D_{n}=\pi_{n}\left(1-\frac{a+a^{-1}}{2}\right)
$$

of the discrete Laplacian on the Schreier graph $\Gamma_{n}$ of $G$. Note that $1-\frac{a+a^{-1}}{2}$ is a non-negative element of the group algebra, since $(1-a)(1-a)^{*}=(1-a)\left(1-a^{-1}\right)=$ $2-a-a^{-1}$. Consequently, the matrices $D_{n}$ are non-negative.

We have

$$
\psi\left(1-\frac{a+a^{-1}}{2}\right)=\left(\begin{array}{cc}
1 & -1 / 2-a / 2 \\
-1 / 2-a^{-1} / 2 & 1
\end{array}\right),
$$

hence

$$
D_{n+1}=\left(\begin{array}{cc}
\pi_{n}(1) & \pi_{n}(-1 / 2-a / 2) \\
\pi_{n}\left(-1 / 2-a^{-1} / 2\right) & \pi_{n}(1)
\end{array}\right) .
$$

It follows that the trace of the form $\mathcal{E}_{n+1}$ on $V_{n}$ is given by the matrix

$$
\begin{aligned}
& \pi_{n}(1)-\pi_{n}(-1 / 2-a / 2) \pi_{n}(1)^{-1} \pi_{n}\left(-1 / 2-a^{-1} / 2\right) \\
& =\pi_{n}\left(1-\frac{1}{4}\left(2+a+a^{-1}\right)\right)=\frac{1}{2} \pi_{n}\left(1-\frac{a+a^{-1}}{2}\right)=\frac{1}{2} D_{n} .
\end{aligned}
$$

We see that the trace of $\mathcal{E}_{n+1}$ on $V_{n}$ is equal to $\frac{1}{2} \mathcal{E}_{n}$. Consequently, if we take the forms $\widetilde{\mathcal{E}}_{n}=2^{n} \mathcal{E}_{n}$, then the trace of $\widetilde{\mathcal{E}}_{n+1}$ on $V_{n}$ is $\widetilde{\mathcal{E}}_{n}$ and we can use Theorem 4.2 to pass to the limit

$$
\mathcal{E}(f, f)=\lim _{n \rightarrow \infty} \widetilde{\mathcal{E}}_{n}(f, f),
$$


where $f$ is a function on the set $V_{*}=\bigcup_{n>0} V_{n}$ of binary rational points on the circle $\mathbb{R} / \mathbb{Z}$ (or, restriction of a function $f \in \bar{C}\left(\mathbb{R} / \mathbb{Z}\right.$ ) onto $V_{*}$ ). Here on the right-hand side of the equality $\widetilde{\mathcal{E}}_{n}$ is evaluated on the restriction of $f$ onto $V_{n}$.

The corresponding finite-dimensional Laplacians are given by the condition

$$
\widetilde{\mathcal{E}}_{n}(f, f)=-\left\langle f \mid \Delta_{n} f\right\rangle_{n}
$$

where $\left\langle f_{1} \mid f_{2}\right\rangle_{n}$ is the normalized inner product

$$
\left\langle f_{1} \mid f_{2}\right\rangle_{n}=\frac{1}{|\mathbf{X}|^{n}} \sum_{v \in \mathbf{X}^{n}} \overline{f_{1}(w v)} f_{2}(w v) .
$$

Consequently, the Laplacians are given by the matrices $-2^{2 n} \pi_{n}\left(1-\frac{a+a^{-1}}{2}\right)$.

Note that for a function $f \in \ell^{2}\left(V_{n}\right)$ given by its values on the points $\frac{k}{2^{n}}$ of the circle, the value of the function $\pi_{n}\left(1-\frac{a+a^{-1}}{2}\right)(f)$ on $t=\frac{k}{2^{n}}$ is equal to

$$
f\left(\frac{k}{2^{n}}\right)-\frac{\frac{k+1}{2^{n}}+\frac{k-1}{2^{n}}}{2}=f(t)-\frac{f\left(t+1 / 2^{n}\right)+f\left(t-1 / 2^{n}\right)}{2} .
$$

Consequently,

$$
\Delta_{n}(f)(t)=-\frac{f(t)-\frac{f\left(t+1 / 2^{n}\right)+f\left(t-1 / 2^{n}\right)}{2}}{1 / 2^{2 n}}
$$

which goes to $f^{\prime \prime}(t)$ as $n \rightarrow \infty$, hence our construction has recovered the classical Laplacian $\Delta(f)=f^{\prime \prime}$ on the circle $\mathbb{R} / \mathbb{Z}$.

5.2. Self-similar random walks. Let us formalize the situation of the last example into a definition. Let $(G, \mathrm{X})$ be a contracting self-similar group. Let $M \in \ell^{1}(G)$ be a Markov operator on $G$, i.e., a linear combination $M=\sum_{g \in G} \mu(g) g$, where $\mu: G \longrightarrow[0, \infty)$ is such that $\sum_{g \in G} \mu(g)=1$. This means that $\mu$ is a probability measure on $G$. We assume that $M$ is symmetric in the sense that $\mu\left(g^{-1}\right)=\mu(g)$. We also assume that the support of $\mu$, which is the set of elements $g$ such that $\mu(g)>0$, generates the whole group $G$. The operator $M$ defines a random walk on $G$, where $\mu(g)$ is the probability of transition from $h$ to $h g$. The corresponding Laplacian is $\Delta=M-1$, where 1 stands for identity operator. If $\mu$ is finitely supported, then $\Delta$ is the discrete Laplacian on the Cayley graph of $G$ and $\pi_{n}(\Delta)$ are the discrete Laplacians on the Schreier graphs $\Gamma_{n}$ of the action of $G$ on the levels $\mathbf{X}^{n}$ of the tree $\mathbf{X}^{*}$.

Consider the Dirichlet forms $\mathcal{E}_{n}$ on $\ell^{2}\left(\mathrm{X}^{n}\right)$ given by the matrices $\pi_{n}(1-M)=$ $-\pi_{n}(\Delta)$. Choose a letter $x_{0} \in \mathbf{X}$, let $w=\ldots x_{0} x_{0} \in \mathbf{X}^{-\omega}$ and let $V_{n} \subset \mathcal{J}_{G}$ be the set of points of the limit space represented by the sequences of the form $w v$ for $v \in \mathrm{X}^{n}$. We consider $\mathcal{E}_{n}$ to be the forms on $V_{n}$, identifying $v \in \mathrm{X}^{n}$ with the corresponding point $w v$ of $V_{n}$.

Then the trace of $\mathcal{E}_{n+1}$ on $V_{n}$ is given by the Schur complement

$$
-\pi_{n}\left(\Delta_{x_{0}, x_{0}}-\Delta_{x_{0}, \overline{x_{0}}}\left(\Delta_{\overline{x_{0}}, \overline{x_{0}}}\right)^{-1} \Delta_{\overline{x_{0}}, x_{0}}\right),
$$

where $\Delta_{x_{0}, x_{0}}$ is the diagonal entry of $\psi(\Delta)$ corresponding to the letter $x_{0}$, and $\Delta_{x_{0}, \overline{x_{0}}}, \Delta_{\overline{x_{0}}, \overline{x_{0}}}$, and $\left.\Delta_{\overline{x_{0}}, x_{0}}\right)$ are the other parts of the matrix $\psi(\Delta)$, so that we have (if we assume that $x_{0}$ is the first letter in the ordering of $\mathbf{X}$ )

$$
\psi(\Delta)=\psi(1-M)=\left(\begin{array}{c|c}
\Delta_{x_{0}, x_{0}} & \Delta_{x_{0}, \overline{x_{0}}} \\
\hline \Delta_{\overline{x_{0}}, x_{0}} & \Delta_{\overline{x_{0}}, \overline{x_{0}}}
\end{array}\right) .
$$


Definition 5.1. The Markov operator $M \in \ell^{1}(G)$ (or the corresponding random walk) is called self-similar if the Schur complement

$$
\Delta^{\prime}=\Delta_{x_{0}, x_{0}}-\Delta_{x_{0}, \overline{x_{0}}}\left(\Delta_{\overline{x_{0}}, \overline{x_{0}}}\right)^{-1} \Delta_{\overline{x_{0}}, x_{0}}
$$

is equal to $\lambda \Delta$ for some $\lambda<1$.

The Schur complement $\Delta^{\prime}$ is equal to $1-M^{\prime}$, where $M^{\prime}$ is a the Markov operator of a naturally defined trace onto $x_{0} \cdot G$ of the random walk on the set X. $G$ defined by $M$. For more details, see [Kai05, GN07, BKN08].

The following theorem is proved in [Kai05]. It is based on a proof of amenability of the Basilica group IMG $\left(z^{2}-1\right)$ given in [BV05].

THEOREM 5.1. If there exists a non-degenerate symmetric self-similar random walk on $G$ having finite entropy, then the group $G$ is amenable.

Here a random walk is said to be non-degenerate, if the support of the corresponding measure $\mu$ generates $G$.

In our setting, if $M$ is a self-similar Markov operator, then the sequence of Dirichlet forms $\widetilde{\mathcal{E}}_{n}$ given by the matrices $\lambda^{-n} \pi_{n}(M-1)$ satisfies the conditions of Theorem 4.2 and thus can be used to construct a Laplacian on the limit space. Note also that this approach is similar to probabilistic constructions of the self-similar diffusions (see, for intance, [Bar98, BB99g, Kus89]).

It has to be noted, that there seems to be not so many examples of self-similar groups with self-similar measures of finite support (see [Kai05] for essentially all known examples). On the other hand, we still do not have any examples of infinitely supported self-similar measures. This makes a direct application of the above theorem complicated. It is still not known if all contracting self-similar groups are amenable.

5.3. Basilica. Let us illustrate the use of self-similar random walks for the case of the Basilica group IMG $\left(z^{2}-1\right)$. Amenability of this group was for the first time proved by B. Virag and L. Bartholdi in [BV05], where self-similar measures appeared for the first time.

Recall, that IMG $\left(z^{2}-1\right)$ is generated by two elements $a, b$, defined by the matrix recursion

$$
\psi(a)=\left(\begin{array}{cc}
0 & b \\
1 & 0
\end{array}\right), \quad \psi(b)=\left(\begin{array}{cc}
1 & 0 \\
0 & a
\end{array}\right)
$$

The limit space of IMG $\left(z^{2}-1\right)$ is homeomorphic to the Julia set of $z^{2}-1$.

Consider a Markov operator of the form $M=\alpha\left(a+a^{-1}\right)+\beta\left(b+b^{-1}\right)$, where $\alpha, \beta>0$ are such that $2(\alpha+\beta)=1$. We have

$$
\psi(1-M)=\left(\begin{array}{cc}
1-2 \beta & -\alpha(1+b) \\
-\alpha\left(1+b^{-1}\right) & 1-\beta\left(a+a^{-1}\right)
\end{array}\right) .
$$


We see that it is convenient to take the second letter as our $x_{0}$, so that the corresponding Schur complement is

$$
\begin{gathered}
1-\beta\left(a+a^{-1}\right)-\left(-\alpha\left(1+b^{-1}\right)\right)(1-2 \beta)^{-1}(-\alpha(1+b))= \\
1-\beta\left(a+a^{-1}\right)-\frac{\alpha^{2}\left(1+b^{-1}\right)(1+b)}{1-2 \beta}= \\
1-\beta\left(a+a^{-1}\right)-\frac{\alpha}{2}\left(2+b+b^{-1}\right)= \\
\left(1-\frac{\alpha}{2}\right)-\left(\beta\left(a+a^{-1}\right)+\frac{\alpha}{2}\left(b+b^{-1}\right)\right) .
\end{gathered}
$$

Consequently, if we want to have a self-similar Markov operator with coefficient $\lambda$, we must have

$$
\beta=\lambda \alpha, \quad \alpha / 2=\lambda \beta,
$$

which gives us the solution $\lambda=1 / \sqrt{2}$ and $\alpha$ and $\beta$ such that $\alpha=\beta \sqrt{2}$. Since $\alpha+\beta=1 / 2$, we get $\alpha=\frac{2-\sqrt{2}}{2}$ and $\beta=\frac{\sqrt{2}-1}{2}$.

It follows that we can take the Dirichlet forms $\mathcal{E}_{n}$ given by the matrices

$$
2^{n / 2} \pi_{n}\left(1-\frac{2-\sqrt{2}}{2}\left(a+a^{-1}\right)-\frac{\sqrt{2}-1}{2}\left(b+b^{-1}\right)\right)
$$

to construct the form $\mathcal{E}$ on the Julia set of $z^{2}-1$.

Next, we describe briefly how the same result can be obtained via analysis on the Julia set of $z^{2}-1$ (called "Basilica", see [Mil99]), which we denote here by $\mathcal{J}$. First, we decompose it as a union

$$
\mathcal{J}=\mathcal{J}_{a} \cup \mathcal{J}_{b}
$$

where

and

$$
\mathcal{J}_{a}=\mathcal{J} \cap\left\{z: \operatorname{Re}(z) \leqslant-\frac{1-\sqrt{5}}{2}\right\}
$$

$$
\mathcal{J}_{b}=\mathcal{J} \cap\left\{z: \operatorname{Re}(z) \geqslant-\frac{1-\sqrt{5}}{2}\right\}
$$

Note that $-\frac{1-\sqrt{5}}{2}$ is the left fixed point of $z^{2}-1$ and, informally, $\mathcal{J}_{a}$ is the left big wing of $\mathcal{J}$ while $\mathcal{J}_{b}$ is the union of the right big wing of $\mathcal{J}$ and the central part of $\mathcal{J}$ (see Figure 4, where the dotted line separates $\mathcal{J}_{a}$ from $\mathcal{J}_{b}$ ).

The polynomial $z^{2}-1$ maps $\mathcal{J}_{a}$ onto $\mathcal{J}_{b}$ by an one-to-one mapping, and also it maps the right wing of $\mathcal{J}_{b}$ onto $\mathcal{J}_{b}$ by a one-to-one mapping. The polynomial $z^{2}-1$ maps the central part of $\mathcal{J}_{b}$ onto $\mathcal{J}_{a}$ by a two-to-one mapping. Therefore the following directed graph

$$
\mathcal{J}_{a} \mathcal{J}_{b} \supset
$$

corresponds to the action of the polynomial $z^{2}-1$ and defines a graph directed cell structure on $\mathcal{J}$. Note that this is related to the following substitution scheme, which gives an inductive rule to construct the Schreier graphs of the action of IMG $\left(z^{2}-1\right)$ on the levels of the tree.
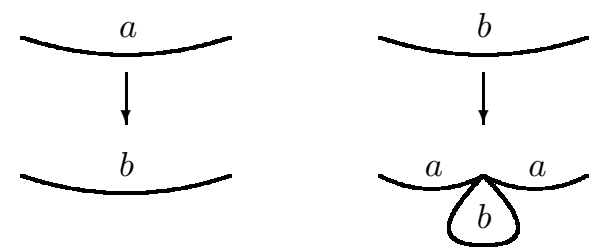
One can see that in two steps a single resistance $a$ is replaced by two resistances $a$, which implies $\rho^{2}=2$ or $\rho=\sqrt{2}$. Since $a$ is replaced by a single $b$ in one step, one finds again that $\alpha=\beta \sqrt{2}$. A background on self-similar fractals in graph directed setting can be found in [HMT06, Met04, HN03]. Although the Basilica Julia set is not self-similar according to these papers, the general method applies, as shown in [RT08]. In particular, one can show that the method of [HN03, KL93] allows to find the spectral dimension $d_{s}$ of the conformally invariant of the Laplacian on the Basilica Julia set by finding such $s$ that the spectral radius of the matrix

$$
(2 \sqrt{2})^{-s}\left(\begin{array}{ll}
0 & 2 \\
1 & 1
\end{array}\right)
$$

is equal to one. This implies $s=\frac{2}{3}$ and $d_{s}=2 s=\frac{4}{3}$. The matrix encodes the substitution rules given above, and also the resistance and measure scaling factors.

5.4. Sierpiński gasket. Similarly to the case of the circle, we begin with the approach of Subsection 4.3 and the Schur complement formula (4.2). The Sierpiński gasket is a union of three contractions of itself, which means that $V_{0}$ consists of the three corner points, and $V_{1}$ consists of six points. Then (4.4) reduces to the matrix equation

$$
\begin{gathered}
\frac{1}{\rho}\left(\begin{array}{rrr}
2 & -1 & -1 \\
-1 & 2 & -1 \\
-1 & -1 & 2
\end{array}\right)= \\
\left(\begin{array}{lll}
2 & 0 & 0 \\
0 & 2 & 0 \\
0 & 0 & 2
\end{array}\right)-\left(\begin{array}{rrr}
-1 & -1 & 0 \\
-1 & 0 & -1 \\
0 & -1 & -1
\end{array}\right)\left(\begin{array}{rrr}
4 & -1 & -1 \\
-1 & 4 & -1 \\
-1 & -1 & 4
\end{array}\right)^{-1}\left(\begin{array}{rrr}
-1 & -1 & 0 \\
-1 & 0 & -1 \\
0 & -1 & -1
\end{array}\right)
\end{gathered}
$$

which implies $\rho=\frac{5}{3}$. Note again that this is a particular case of Proposition 4.9. This construction coincides with the classical construction of the Laplacian on the Sierpiński gasket originally due to [BP88, Kig89, Kus87, Kus89].

Recall that the Sierpiński gasket is homeomorphic to the limit space of the Hanoi tower group $H_{3}$ (see 2.7.2). The generators $a_{0}, a_{1}, a_{2}$ of $H_{3}$ satisfy the matrix recursions

$$
\psi\left(a_{0}\right)=\left(\begin{array}{ccc}
a_{0} & 0 & 0 \\
0 & 0 & 1 \\
0 & 1 & 0
\end{array}\right), \quad \psi\left(a_{1}\right)=\left(\begin{array}{ccc}
0 & 0 & 1 \\
0 & a_{1} & 0 \\
1 & 0 & 0
\end{array}\right), \quad \psi\left(a_{2}\right)=\left(\begin{array}{ccc}
0 & 1 & 0 \\
1 & 0 & 0 \\
0 & 0 & a_{2}
\end{array}\right) .
$$

Consider set of sequences $W=\left\{w_{0}=\ldots 000, w_{1}=\ldots 111, w_{2}=\ldots 222\right\}$ and the corresponding representations $\rho=\rho_{W}$ of $C\left(\mathcal{J}_{G}\right)$ and $\pi=\pi_{W}$ of $\mathbb{C} G$. We have $w_{i} x_{i}=w_{i}$ for $x_{0}=0, x_{1}=1, x_{2}=2$.

It follows from the structure of the nucleus of the Hanoi tower group $G$ that sequences $i^{-\omega} j v=\ldots i i i j v$ and $i^{-\omega} k v=\ldots i i i k v$, for any $v \in \mathrm{X}^{*}$ and any permutation $(i, j, k)$ of $0,1,2$, define the same points of the limit space $\mathcal{J}_{G}$. Let $\ell^{2}\left(V_{n}\right)$ be the subspace of $\ell^{2}\left(W \mathrm{X}^{n}\right)$ consisting of the functions having equal values on equivalent sequences.

Let us take the element

$$
D(x, y)=\left(\begin{array}{ccc}
y\left(1-a_{0}\right)+2 x & -x & -x \\
-x & y\left(1-a_{1}\right)+2 x & -x \\
-x & -x & y\left(1-a_{2}\right)+2 x
\end{array}\right)
$$

of the algebra $M_{3 \times 3}(\mathbb{C} G)$, where $x$ and $y$ are positive real numbers. 
Let $\mathcal{E}_{n, x}$ be the form on $\ell^{2}\left(W \mathrm{X}^{n}\right)$ given by the matrix $\pi_{n}(D(x, y))$. We see that $\psi(D(x, y))$ is

$\left(\begin{array}{ccc|ccc|ccc}y \widetilde{a_{0}}+2 x & 0 & 0 & -x & 0 & 0 & -x & 0 & 0 \\ 0 & y+2 x & -y & 0 & -x & 0 & 0 & -x & 0 \\ 0 & -y & y+2 x & 0 & 0 & -x & 0 & 0 & -x \\ \hline-x & 0 & 0 & y+2 x & 0 & -y & -x & 0 & 0 \\ 0 & -x & 0 & 0 & y \widetilde{a}_{1}+2 x & 0 & 0 & -x & 0 \\ 0 & 0 & -x & -y & 0 & y+2 x & 0 & 0 & -x \\ \hline-x & 0 & 0 & -x & 0 & 0 & y+2 x & -y & 0 \\ 0 & -x & 0 & 0 & -x & 0 & -y & y+2 x & 0 \\ 0 & 0 & -x & 0 & 0 & -x & 0 & 0 & y \widetilde{a}_{2}+2 x\end{array}\right)$,

where $\widetilde{a}_{i}=1-a_{i}$.

Let us permute the rows and columns (permute the basis) so that in the left top corner we get the action on the subspace $\ell^{2}\left(W \mathrm{X}^{n}\right)$ of $\ell^{2}\left(W^{n+1}\right)$ :

$\left(\begin{array}{ccc|cccccc}y \widetilde{a}_{0}+2 x & 0 & 0 & -x & 0 & 0 & -x & 0 & 0 \\ 0 & y \widetilde{a}_{1}+2 x & 0 & 0 & -x & 0 & 0 & -x & 0 \\ 0 & 0 & y \widetilde{a}_{2}+2 x & 0 & 0 & -x & 0 & 0 & -x \\ \hline-x & 0 & 0 & 1+2 x & 0 & -1 & -x & 0 & 0 \\ 0 & -x & 0 & 0 & y+2 x & 0 & 0 & -x & -y \\ 0 & 0 & -x & -y & 0 & y+2 x & 0 & 0 & -x \\ -x & 0 & 0 & -x & 0 & 0 & y+2 x & -y & 0 \\ 0 & -x & 0 & 0 & -x & 0 & -y & y+2 x & 0 \\ 0 & 0 & -x & 0 & -y & -x & 0 & 0 & y+2 x\end{array}\right)$.

The trace of $\mathcal{E}_{n+1, x, y}$ on the subspace $\ell^{2}\left(W \mathrm{X}^{n}\right)$ of $\ell^{2}\left(W \mathrm{X}^{n+1}\right)$ is then the Schur complement of the top left corner of the partitioned matrix above. A direct computation shows that it is equal to

$$
D\left(\frac{3 x y}{3 x+5 y}, y\right)=D\left(\frac{3}{5+\frac{3 x}{y}} x, y\right) .
$$

Recall that $\ell^{2}\left(V_{n}\right)$ is the subspace of $\ell^{2}\left(W \mathrm{X}^{n}\right)$ consisting of the functions constant on the asymptotic equivalence classes. It follows from the description of the equivalence classes on $\mathrm{X}^{-\omega}$ associated with $H_{3}$ that the values of $\mathcal{E}_{n, x, y}$ on $\ell^{2}\left(V_{n}\right)$ do not depend on $y$. Let $\mathcal{E}_{n, x}^{V}$ be the restriction of $\mathcal{E}_{n, x, y}$ onto $\ell^{2}\left(V_{n}\right)$. One can show then that $\operatorname{Trace}_{V_{n}} \mathcal{E}_{n+1, x}^{V}$ is equal to the restriction of

$$
\lim _{y \rightarrow \infty} \operatorname{Trace}_{W \mathbf{X}^{n}} \mathcal{E}_{n+1, x, y}
$$

onto $\ell^{2}\left(V_{n}\right)$. Letting $y$ go to infinity corresponds to gluing together equivalent points of $W \mathrm{X}^{n}$ (making conductance between them infinite).

We get then $\operatorname{Trace}_{V_{n}} \mathcal{E}_{n+1, x}^{V}=\frac{3}{5} \mathcal{E}_{n, x}^{V}$. Consequently, we can construct a Dirichlet form (hence a Laplacian) on the Sierpiński gasket $\mathcal{J}_{H_{3}}$ as passing to the limit of the sequence of the Dirichlet forms $\left(\frac{5}{3}\right)^{n} \mathcal{E}_{n, x}^{V}$.

The above computations of the Schur complement in the Hanoi tower group appears for the first time in a computation of the spectra of its Schreier graph by R. Grigorchuk and Z. Šunić [GŠ08].

5.5. Strictly p.c.f. groups. The Hanoi tower group is strictly p.c.f. (see Definition 4.2), which makes the standard technique of analysis on finitely ramified fractals applicable.

Suppose that $(G, X)$ is a strictly p.c.f. finitely generated self-replicating group. It is generated then by its nucleus $\mathcal{N}$.

Let $W$ be the set of infinite sequences $w=\ldots x_{2} x_{1}$ such that there exists an oriented path $\ldots e_{2} e_{1}$ in the Moore diagram of the nucleus ending in a non-trivial element and such that the arrow $e_{n}$ is labeled by $\left(x_{n}, y_{n}\right)$ for some $y_{n}$. Then it 
follows from the definition of strictly p.c.f. groups that $y_{n}=x_{n}$. Let us denote by $g_{w} \in \mathbb{C} G$ the average of the elements of $G$ corresponding to the ends of paths $\ldots e_{2} e_{1}$ with labels $\ldots\left(x_{2}, x_{2}\right)\left(x_{1}, x_{1}\right)$. One can prove that the set of such elements is a finite group (the isotropy group of $w$, see [Nek05]).

Recall that by Proposition 2.8, the set $W$ is precisely the set of sequences $w$ such that for some $v$ the point represented by $w v$ belongs to the boundary of the tile $\mathcal{T}_{v}$.

Consider now the symmetric matrix $D(\vec{x}, y)=\left(D_{w_{1}, w_{2}}\right) \in M_{W \times W}(\mathbb{C} G)$, for

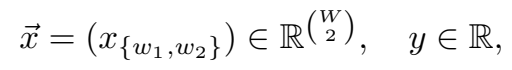

with the elements

$$
D_{w_{1}, w_{2}}=-x_{\left\{w_{1}, w_{2}\right\}},
$$

if $w_{1} \neq w_{2}$, and

$$
D_{w, w}=y\left(1-g_{w}\right)+\sum_{w_{2} \neq w} x_{\left\{w, w_{2}\right\}} .
$$

One can prove that trace of the quadratic form with matrix $D(\vec{x}, y)$ on the subspace $\ell^{2}(W)$ of $\ell^{2}(W \mathrm{X})$ is the quadratic form with matrix $D(R(\vec{x}, y))$ for some rational function $R$. Rather than giving a proof of this statement, we will consider another example of a strictly p.c.f. group below.

Note that here, as in the case of the Hanoi tower group, values of the quadratic form with the matrix $\pi_{n}(D(\vec{x}, y))$ on the subspace $\ell^{2}\left(V_{n}\right)$ of $\ell^{2}\left(W \mathrm{X}^{n}\right)$ does not depend on $y$, so that we may also pass to the limit as $y \rightarrow \infty$.

In this way we reduce the question of construction of a self-similar Dirichlet form to a non-linear finite-dimensional eigenvector problem. If the limit space has additional symmetries, it may be possible to reduce the number of variables setting some of coordinates of $\vec{x}$ equal. This is, essentially, the eigenvalue problem (4.4).

5.6. Pillow. In contrast to the cases of the circle and the Sierpiński gasket, Proposition 4.9 does not apply to the Pillow example. The approach of Subsection 4.3 requires to solve the nonlinear eigenvalue equation (4.4), which is not easy to do in this case. Proposition 4.10 implies that there is a unique, up to a constant multiple, self-similar regular Dirichlet form. Note that the cone $\mathbb{D}$ is six-dimensional and the cone $\mathbb{D}_{\mathfrak{G}}$ is three-dimensional. Its boundary consists of Dirichlet forms that have at least two zero conductances. Then it is easy to verify that there are no eigenvectors of $\Lambda$ on the boundary of $\mathbb{D}_{\mathfrak{G}}$ by exhausting all possible choices. Moreover, it is not difficult to see that $\rho_{1}=\rho_{2}>1$, and so the Dirichlet form is regular.

The method to obtain existence and uniqueness in the above paragraph does not help in finding the eigenvector of $\Lambda$. However, one still can successfully apply the same strategy as for the other strictly p.c.f. examples. Consider the group generated by $a, b, c$, which are given by the matrix recursion

$$
a \mapsto\left(\begin{array}{cc}
0 & 1 \\
1 & 0
\end{array}\right), \quad b \mapsto\left(\begin{array}{cc}
a & 0 \\
0 & a
\end{array}\right), \quad c \mapsto\left(\begin{array}{cc}
b & 0 \\
0 & c
\end{array}\right) .
$$

It is a strictly p.c.f. group with the limit space shown on Figure 7.

We see from the recursion that the set $W$ of sequences read on paths ending in non-trivial states of the nucleus is

$$
W=\left\{w_{0}=1^{-\omega} 00, w_{1}=1^{-\omega} 01, w_{2}=1^{-\omega} 0, w_{3}=1^{-\omega}\right\} .
$$




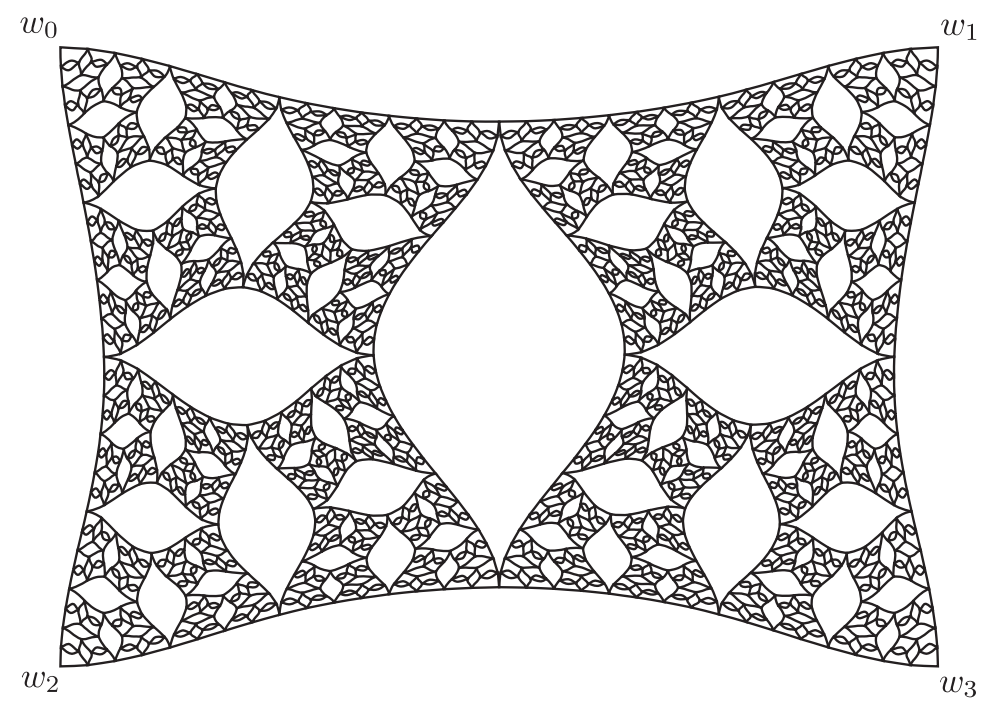

FiguRe 7. Pillow fractal

We have $g_{w_{0}}=a, g_{w_{1}}=a, g_{w_{2}}=b, g_{w_{3}}=c$. The sequences $w_{i}$ represent the "corner" points of the pillow, as it is shown on Figure 7 . The sequences $w_{i} v$ represent then the corners of the corresponding tiles $\mathcal{T}_{v}$ (every point of the boundary of $\mathcal{T}_{v}$ is such a corner).

The shift s : $\mathcal{J}_{G} \longrightarrow \mathcal{J}_{G}$ folds the limit space in two and then rotates it by $\pi / 2$, so that $\mathbf{s}\left(w_{0}\right)=w_{2}, \mathbf{s}\left(w_{1}\right)=w_{2}, \mathbf{s}\left(w_{2}\right)=w_{3}$ and $\mathbf{s}\left(w_{3}\right)=w_{3}$.

Symmetries of the pillow fractal suggest that we may try to set $x_{\left\{w_{0}, w_{1}\right\}}=$ $x_{\left\{w_{2}, w_{3}\right\}}, x_{\left\{w_{0}, w_{2}\right\}}=x_{\left\{w_{1}, w_{3}\right\}}$ and $x_{\left\{w_{0}, w_{3}\right\}}=x_{\left\{w_{1}, w_{2}\right\}}$.

So, we take $D(p, q, r, y)$ to be

$$
\left(\begin{array}{cccc}
y(1-a)+s & -p & -q & -r \\
-p & y(1-a)+s & -r & -q \\
-q & -r & y(1-b)+s & -p \\
-r & -q & -p & y(1-c)+s
\end{array}\right)
$$

where $s=p+q+r$.

Application of the matrix recursion gives

$$
\left(\begin{array}{cccc|cccc}
y+s & -y & -p & 0 & -q & 0 & -r & 0 \\
-y & y+s & 0 & -p & 0 & -q & 0 & -r \\
-p & 0 & y+s & -y & -r & 0 & -q & 0 \\
0 & -p & -y & y+s & 0 & -r & 0 & -q \\
\hline-q & 0 & -r & 0 & y \widetilde{a}+s & 0 & -p & 0 \\
0 & -q & 0 & -r & 0 & y \widetilde{a}+s & 0 & -p \\
-r & 0 & -q & 0 & -p & 0 & y \widetilde{b}+s & 0 \\
0 & -r & 0 & -q & 0 & -p & 0 & y \widetilde{c}+s
\end{array}\right),
$$

where $\widetilde{a}=1-a, \widetilde{b}=1-b$ and $\widetilde{c}=1-c$.

Computing the Schur complement and letting $y$ go to infinity, we see that the trace on $V_{n}$ of the form defined by $\pi_{n+1}(D(p, q, r, y))$ on $\ell^{2}\left(V_{n+1}\right)$ is the form defined 
on $V_{n}$ by the matrix $\pi_{n}\left(D\left(p^{\prime}, q^{\prime}, r^{\prime}, y\right)\right)$, where

$$
p^{\prime}=\frac{p(q+r)+q^{2}+r^{2}}{2(2 p+q+r)}, \quad q^{\prime}=p+\frac{p(q+r)+2 q r}{2(2 p+q+r)}, \quad r^{\prime}=\frac{p(q+r)+2 q r}{2(2 p+q+r)} .
$$

Note that in computation of the Schur complement only the coefficients at $y^{2}$ have to be computed, since $y^{4}$ and $y^{3}$ cancel and the lower terms are not important when passing to the limit as $y \rightarrow \infty$. This observation substantially simplifies the computations.

One can see by direct but lengthy computation that solving nonlinear eigenvalue problem (4.4) leads to the same equations.

\section{Analysis and probability on non p.c.f. fractals}

6.1. On the relation between p.c.f. and finitely ramified assumptions, and self-similar metrics. A compact connected metric space $\mathcal{X}$ is called a finitely ramified self-similar set if there are injective contraction maps

$$
\psi_{0}, \ldots, \psi_{d-1}: \mathcal{X} \rightarrow \mathcal{X}
$$

and a finite set $V_{0} \subset \mathcal{X}$ such that

$$
\mathcal{X}=\bigcup_{i=0}^{d-1} \psi_{i}(\mathcal{X})
$$

and for any $n$ and for any two distinct words $w, w^{\prime} \in \mathbf{X}^{n}=\{1, \ldots, d\}^{n}$ we have

$$
\mathcal{X}_{w} \cap \mathcal{X}_{w^{\prime}}=V_{w} \cap V_{w^{\prime}}
$$

where $\mathcal{X}_{w}=\psi_{w}(\mathcal{X})$ and $V_{w}=\psi_{w}\left(V_{0}\right)$. Here for a finite word $w=w_{1} \ldots w_{n} \in \mathbf{X}^{n}$ we denote

$$
\psi_{w}=\psi_{w_{1}} \circ \cdots \circ \psi_{w_{n}}
$$

The set $V_{0}$ is called the vertex boundary of $\mathcal{X}$, and $\mathcal{X}_{w}$ are called cells.

The question of existence of a "self-similar" metric on self-similar sets was recently studied in detail in [Hve05, Kam00, Kam04]. According to [Hve05], our class of self-similar finitely ramified fractals defined above is the same as finitely ramified SSH-fractals (with finite fractal boundary) of [Hve05]. The definition of SSH-fractals in [Hve05] requires fulfillment of a certain set of axioms, one of which is that the maps $\psi_{0}, \ldots, \psi_{d-1}: \mathcal{X} \rightarrow \mathcal{X}$ are continuous injections. It is then proved that $\mathcal{X}$ can be equipped with a self-similar metric in such a way that the injective maps $\psi_{j}$ become contractions (as well as local similitudes), but the topology does not change. In particular, for every p.c.f. self-similar set defined in [Kig01, Kig03] there exists a self-similar metric. Therefore the definition of self-similar finitely ramified fractals generalizes the definition of p.c.f. self-similar sets. The definition of a finitely ramified set allows infinitely many cells with disjoint interiors to meet at a junction point, which is referred to as fractals with "infinite multiplicity" in [Hve05]. We use a simplified approach when we assume from the very beginning that $\psi_{i}$ are contractions. A simple example of self-similar finitely ramified fractal which is not a p.c.f. self-similar set is the Diamond fractal shown in Figure 8. Recently this fractal was studied in [HK08] in relation to the critical percolation and in $\left[\mathbf{B C D}^{+} \mathbf{0 8}\right]$ in relation to the self-similarity of the spectrum and spectral decimation. 


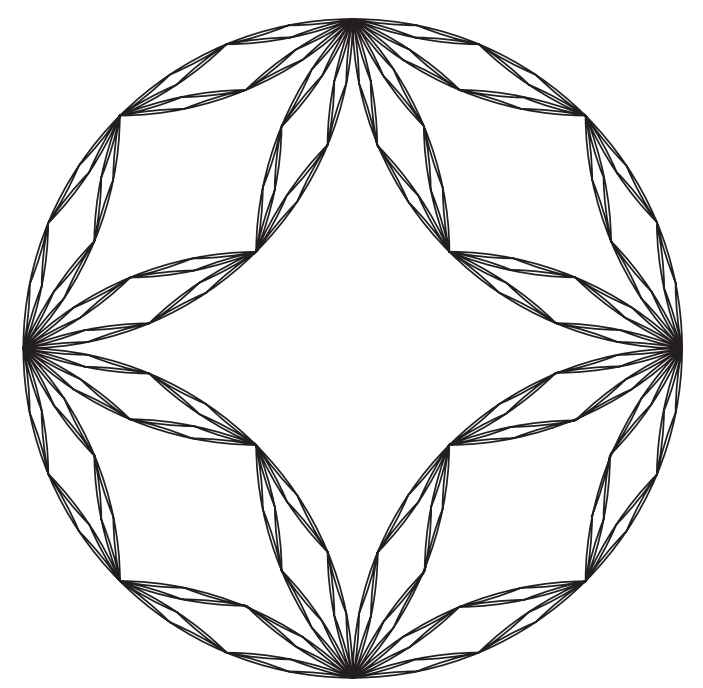

FiguRE 8. The diamond fractal, a finitely ramified non p.c.f. selfsimilar set.

An $n$-cell is called a boundary cell if it intersects $V_{0}$. Otherwise it is called an interior cell. We say that $\mathcal{X}$ has connected interior if the set of interior 1cells is connected, any boundary 1-cell contains exactly one point of $V_{0}$, and the intersection of two different boundary 1-cells is contained in an interior 1-cell. The following theorem is proved in [HMT06] for the p.c.f. case, but the proof applies for self-similar finitely ramified fractal without any changes.

THEOREM 6.1. Suppose that $\mathcal{X}$ has connected interior, and a group $\mathfrak{G}$ acts on a self-similar finitely ramified fractal $\mathcal{X}$ such that its action on $V_{0}$ is transitive. Then there are weights $\rho_{i}>1$ such that there exists a $\mathfrak{G}$-invariant self-similar resistance form $\mathcal{E}$ with these weights.

6.2. Non finitely ramified fractals. In the case non-p.c.f. ramified case, there is an existence result [BB99] for the so-called generalized Sierpiński carpets (see also [BB89, BB90, BBS90, KY92]), but the question of uniqueness is currently under investigation [BBKT08]. It seems plausible that the developed methods, which are quite complicated, to prove existence (and possibly uniqueness) on the generalized Sierpiński carpets can be adapted for the limit spaces of selfsimilar groups, but it is far from certain. We will not discuss these questions in the present paper, but only mention that they are related to the local symmetries of the space, elliptic and parabolic Harnack inequalities, and to sub-Gaussian type heat kernel (transition probabilities) estimates on metric measure spaces

$$
\frac{1}{t^{\alpha / \beta}} \Phi_{1}\left(\frac{d(x, y)}{t^{1 / \beta}}\right) \leqslant p_{t}(x, y) \leqslant \frac{1}{t^{\alpha / \beta}} \Phi_{2}\left(\frac{d(x, y)}{t^{1 / \beta}}\right) .
$$

One can see see [BB04, BBK06, GHL06, and references therein] for more details. The present volume contains an interesting relevant article [GK08] by A. Grigoryan and T. Kumagai on the dichotomy in the heat kernel two sided estimates. In particular, the dichotomy means drastically different behavior of local and non local Dirichlet forms, which may be related to the questions raised in Subsection 5.2. 


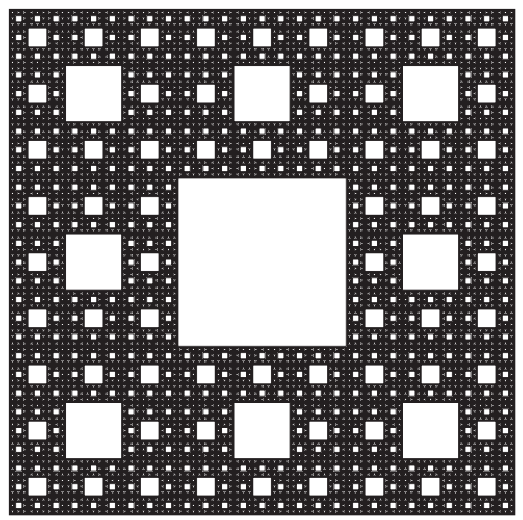

Figure 9. The Sierpiński carpet.

\section{References}

[Ati70] M. F. Atiyah, Global theory of elliptic operators, Proc. Internat. Conf. on Functional Analysis and Related Topics (Tokyo, 1969), Univ. of Tokyo Press, Tokyo, 1970, pp. 2130. MR MR0266247 (42 \#1154)

[Bar98] Martin T. Barlow, Diffusions on fractals, Lectures on probability theory and statistics (Saint-Flour, 1995), Lecture Notes in Math., vol. 1690, Springer, Berlin, 1998, pp. 1121. MR MR1668115 (2000a:60148)

[BB89] Martin T. Barlow and Richard F. Bass, The construction of Brownian motion on the Sierpiński carpet, Ann. Inst. H. Poincaré Probab. Statist. 25 (1989), no. 3, 225-257. MR MR1023950 (91d:60183)

[BB90] Local times for Brownian motion on the Sierpinski carpet, Probab. Theory Related Fields 85 (1990), no. 1, 91-104. MR MR1044302 (91j:60129)

[BB99g] M. T. Barlow and R. F. Bass, Random walks on graphical Sierpinski carpets. Random walks and discrete potential theory (Cortona, 1997), 26-55, Sympos. Math., XXXIX, Cambridge Univ. Press, Cambridge, 1999.

[BB99] Brownian motion and harmonic analysis on Sierpinski carpets, Canad. J. Math. 51 (1999), no. 4, 673-744. MR MR1701339 (2000i:60083)

[BB04] Stability of parabolic Harnack inequalities, Trans. Amer. Math. Soc. 356 (2004), no. 4, 1501-1533 (electronic). MR MR2034316 (2005e:60167)

[BBK06] Martin T. Barlow, Richard F. Bass, and Takashi Kumagai, Stability of parabolic Harnack inequalities on metric measure spaces, J. Math. Soc. Japan 58 (2006), no. 2, 485-519. MR MR2228569 (2007f:60064)

[BBKT08] Martin T. Barlow, Richard F. Bass, Takashi Kumagai, and Alexander Teplyaev, Uniqueness of brownian motion on sierpinski carpets, preprint (2008).

[BBS90] M. T. Barlow, R. F. Bass, and J. D. Sherwood, Resistance and spectral dimension of Sierpiński carpets, J. Phys. A 23 (1990), no. 6, L253-L258. MR MR1048762 (91a:28007)

$\left[\mathrm{BCD}^{+} 08\right]$ N. Bajorin, T. Chen, A. Dagan, C. Emmons, M. Hussein, M. Khalil, P. Mody, B. Steinhurst, and A. Teplyaev, Vibration modes of 3 n-gaskets and other fractals, J. Phys. A: Math. Theor. 41 (2008), no. 1, 015101 (21pp).

[BDF73] L. G. Brown, R. G. Douglas, and P. A. Fillmore, Extensions of $C^{*}$-algebras, operators with compact self-commutators, and K-homology, Bull. Amer. Math. Soc. 79 (1973), 973-978.

[BG00] Laurent Bartholdi and Rostislav I. Grigorchuk, On the spectrum of Hecke type operators related to some fractal groups, Proceedings of the Steklov Institute of Mathematics 231 (2000), 5-45.

[BGN03] Laurent Bartholdi, Rostislav Grigorchuk, and Volodymyr Nekrashevych, From fractal groups to fractal sets, Fractals in Graz 2001. Analysis - Dynamics - Geometry 
- Stochastics (Peter Grabner and Wolfgang Woess, eds.), Birkhäuser Verlag, Basel, Boston, Berlin, 2003, pp. 25-118.

[BGŠ03] Laurent Bartholdi, Rostislav I. Grigorchuk, and Zoran Šuniḱ, Branch groups, Handbook of Algebra, Vol. 3, North-Holland, Amsterdam, 2003, pp. 989-1112.

[BH91] Nicolas Bouleau and Francis Hirsch, Dirichlet forms and analysis on Wiener space, de Gruyter Studies in Mathematics, vol. 14, Walter de Gruyter \& Co., Berlin, 1991. MR MR1133391 (93e:60107)

[BKN08] Laurent Bartholdi, Vadim Kaimanovich and Volodymyr Nekrashevych, On amenability of automata groups, (preprint arXiv:0802.2837), 2008.

[BN03] Evgen Bondarenko and Volodymyr Nekrashevych, Post-critically finite self-similar groups, Algebra and Discrete Mathematics 2 (2003), no. 4, 21-32.

[BN06] Laurent Bartholdi and Volodymyr V. Nekrashevych, Thurston equivalence of topological polynomials, Acta Math. 197 (2006), no. 1, 1-51.

[BN08] Laurent Bartholdi and Volodymyr V. Nekrashevych, Iterated monodromy groups of quadratic polynomials I, to appear in "Groups, Geometry and Dynamics", 2008.

[BP88] Martin T. Barlow and Edwin A. Perkins, Brownian motion on the Sierpiński gasket, Probab. Theory Related Fields 79 (1988), no. 4, 543-623. MR MR966175 (89g:60241)

[BSV99] Andrew M. Brunner, Said N. Sidki, and Ana. C. Vieira, A just-nonsolvable torsion-free group defined on the binary tree, J. Algebra 211 (1999), 99-144.

[BV05] Laurent Bartholdi and Bálint Virág, Amenability via random walks, Duke Math. J. 130 (2005), no. 1, 39-56.

[Con94] Alain Connes, Noncommutative geometry, San Diego, CA: Academic Press, 1994.

[FŌT94] Masatoshi Fukushima, Yōichi Ōshima, and Masayoshi Takeda, Dirichlet forms and symmetric Markov processes, de Gruyter Studies in Mathematics, vol. 19, Walter de Gruyter \& Co., Berlin, 1994. MR MR1303354 (96f:60126)

[GH90] Étienne Ghys and Pierre de la Harpe, Sur les groupes hyperboliques d'après Mikhael Gromov, Progress in Mathematics, vol. 83, Birkhäuser Boston Inc., Boston, MA, 1990, Papers from the Swiss Seminar on Hyperbolic Group held in Bern, 1988.

[GHL06] Alexander Grigoryan, Jiaxin Hu, and Ka-Sing Lau, Equivalence conditions for ondiagonal upper bounds of heat kernels on self-similar spaces, J. Funct. Anal. 237 (2006), no. 2, 427-445. MR MR2230344 (2007j:60123)

[GK08] Alexander Grigoryan and Takashi Kumagai, On the dichotomy in the heat kernel two sided estimates, Analysis on Graphs and its Applications, Proc. Symp. Pure Math. (this volume), Amer. Math. Soc., 2008.

[GLSŻ00] Rostislav I. Grigorchuk, Peter Linnell, Thomas Schick, and Andrzej Żuk, On a question of Atiyah, C. R. Acad. Sci. Paris Sér. I Math. 331 (2000), no. 9, 663-668.

[GN07] Rostislav Grigorchuk and Volodymyr Nekrashevych, Self-similar groups, operator algebras and Schur complement, Journal of Modern Dynamics 1 (2007), no. 3, 323-370.

[GNS00] Rostislav I. Grigorchuk, Volodymyr V. Nekrashevich, and Vitaliu I. Sushchanskii, Automata, dynamical systems and groups, Proceedings of the Steklov Institute of Mathematics 231 (2000), 128-203.

[Gri80] Rostislav I. Grigorchuk, On Burnside's problem on periodic groups, Functional Anal. Appl. 14 (1980), no. 1, 41-43.

[Gri85] - Degrees of growth of finitely generated groups and the theory of invariant means, Math. USSR Izv. 25 (1985), no. 2, 259-300.

[Gro87] Mikhael Gromov, Hyperbolic groups, Essays in Group Theory (S. M. Gersten, ed.), M.S.R.I. Pub., no. 8, Springer, 1987, pp. 75-263.

[GS83] Narain D. Gupta and Said N. Sidki, On the Burnside problem for periodic groups, Math. Z. 182 (1983), 385-388.

[GS̆06] R. Grigorchuk and Z. Šunić, Asymptotic aspects of Schreier graphs and Hanoi Towers groups, Comptes Rendus Mathématique, Académie des Sciences Paris 342 (2006), no. $8,545-550$.

[GS̆08] R. Grigorchuk and Z. Šunić, Schreier spectrum of the Hanoi Towers group on three pegs, Analysis on Graphs and its Applications, Proc. Symp. Pure Math. (this volume), Amer. Math. Soc., 2008.

[GŻ01] Rostislav I. Grigorchuk and Andrzej Żuk, The lamplighter group as a group generated by a 2-state automaton and its spectrum, Geom. Dedicata 87 (2001), no. 1-3, 209-244. 
[GŻ02a] On a torsion-free weakly branch group defined by a three state automaton, Internat. J. Algebra Comput. 12 (2002), no. 1, 223-246.

[GŻ02b] Spectral properties of a torsion-free weakly branch group defined by a three state automaton, Computational and Statistical Group Theory (Las Vegas, NV/Hoboken, NJ, 2001), Contemp. Math., vol. 298, Amer. Math. Soc., Providence, RI, 2002, pp. 57-82.

[HK08] Ben M. Hambly and Takashi Kumagai, Diffusion on the scaling limits of critical percolation clusters in the diamond hierarchical lattice, preprint (2008).

[HMT06] B. M. Hambly, V. Metz, and A. Teplyaev, Self-similar energies on post-critically finite self-similar fractals, J. London Math. Soc. (2) 74 (2006), no. 1, 93-112. MR MR2254554 (2007i:31011)

[HN03] B. M. Hambly and S. O. G. Nyberg, Finitely ramified graph-directed fractals, spectral asymptotics and the multidimensional renewal theorem, Proc. Edinb. Math. Soc. (2) 46 (2003), no. 1, 1-34. MR MR1961168 (2004a:28014)

[HP06] Peter Haïssinsky and Kevin M. Pilgrim, Coarse expanding conformal dynamics, (preprint, available at arXiv:math/0612617v1), 2006.

[Hve05] Klara Hveberg, Injective mapping systems and self-homeomorphic fractals, Ph.D. thesis, University of Oslo, 2005.

[Kai05] Vadim Kaimanovich, "Münchhausen trick" and amenability of self-similar groups, International Journal of Algebra and Computation 15 (2005), no. 5-6, 907-937.

[Kam00] Atsushi Kameyama, Distances on topological self-similar sets and the kneading determinants, J. Math. Kyoto Univ. 40 (2000), no. 4, 601-672. MR MR1802840 (2001m:37024)

[Kam04] Distances on topological self-similar sets, Fractal geometry and applications: a jubilee of Benoit Mandelbrot. Part 1, Proc. Sympos. Pure Math., vol. 72, Amer. Math. Soc., Providence, RI, 2004, pp. 117-129. MR MR2112104 (2005i:37014)

[Kas75] G. G. Kasparov, Topological invariants of elliptic operators. I. K-homology, Izv. Akad. Nauk SSSR Ser. Mat. 39 (1975), no. 4, 796-838.

[Kig89] Jun Kigami, A harmonic calculus on the Sierpiński spaces, Japan J. Appl. Math. 6 (1989), no. 2, 259-290. MR MR1001286 (91g:31005)

[Kig93] Harmonic calculus on p.c.f. self-similar sets, Trans. Amer. Math. Soc. 335 (1993), no. 2, 721-755. MR MR1076617 (93d:39008)

[Kig01] Analysis on fractals, Cambridge Tracts in Mathematics, vol. 143, Cambridge University Press, Cambridge, 2001. MR MR1840042 (2002c:28015)

[Kig03] , Harmonic analysis for resistance forms, J. Funct. Anal. 204 (2003), no. 2, 399-444. MR MR2017320 (2004m:31010)

[KL93] Jun Kigami and Michel L. Lapidus, Weyl's problem for the spectral distribution of Laplacians on p.c.f. self-similar fractals, Comm. Math. Phys. 158 (1993), no. 1, 93125. MR MR1243717 (94m:58225)

[Kus87] Shigeo Kusuoka, A diffusion process on a fractal, Probabilistic methods in mathematical physics (Katata/Kyoto, 1985), Academic Press, Boston, MA, 1987, pp. 251-274. MR MR933827 (89e:60149)

[Kus89] - Dirichlet forms on fractals and products of random matrices, Publ. Res. Inst. Math. Sci. 25 (1989), no. 4, 659-680. MR MR1025071 (91m:60142)

[KY92] Shigeo Kusuoka and Zhou Xian Yin, Dirichlet forms on fractals: Poincaré constant and resistance, Probab. Theory Related Fields 93 (1992), no. 2, 169-196. MR MR1176724 (94e:60069)

[Lax02] Peter D. Lax, Functional analysis, Pure and Applied Mathematics (New York), WileyInterscience [John Wiley \& Sons], New York, 2002. MR MR1892228 (2003a:47001)

[Lin91] Tom Lindstrøm, Nonstandard analysis, iterated function systems, and Brownian motion on fractals, Contemporary stochastic analysis (Ibadan, 1989), World Sci. Publ., River Edge, NJ, 1991, pp. 71-108. MR MR1230402 (94k:60124)

[McM00] Curtis T. McMullen, Hausdorff dimension and conformal dynamics. II. Geometrically finite rational maps, Comment. Math. Helv. 75 (2000), no. 4, 535-593.

[Met03] Volker Metz, The cone of diffusions on finitely ramified fractals, Nonlinear Anal. 55 (2003), no. 6, 723-738. MR MR2060526 (2005d:60129)

[Met04] , "Laplacians" on finitely ramified, graph directed fractals, Math. Ann. 330 (2004), no. 4, 809-828. MR MR2102313 (2005i:31016) 
[Mil99] John Milnor, Dynamics in one complex variable. Introductory lectures, Wiesbaden: Vieweg, 1999.

[Miš74] A. S. Miščenko, Infinite-dimensional representations of discrete groups, and higher signatures, Izv. Akad. Nauk SSSR Ser. Mat. 38 (1974), 81-106.

[Nek03] Volodymyr Nekrashevych, Iterated monodromy groups, Dopov. Nats. Akad. Nauk Ukr., Mat. Pryr. Tekh. Nauky (2003), no. 4, 18-20, (in Ukrainian).

[Nek05] _ Self-similar groups, Mathematical Surveys and Monographs, vol. 117, Amer. Math. Soc., Providence, RI, 2005.

[Nek07] — Free subgroups in groups acting on rooted trees, (preprint arXiv:0802.2554), 2008.

[Nek08a] Symbolic dynamics and self-similar groups, to appear in "Holomorphic Dynamics and Renormalization, in Honour of John Milnor's 75th Birthday", volume 53 of Fields Communications Series, 2008.

[Nek08b] — $C^{*}$-algebras and self-similar groups, to appear in "Journal für die reine und angewandte Mathematik", 2008.

[Pei06] Roberto Peirone, Convergence of Dirichlet forms on fractals, Topics on concentration phenomena and problems with multiple scales, Lect. Notes Unione Mat. Ital., vol. 2, Springer, Berlin, 2006, pp. 139-188. MR MR2267882

[Pei08] _ Existence of eigenforms on nicely separated fractals, Analysis on Graphs and its Applications, Proc. Symp. Pure Math. (this volume), Amer. Math. Soc., 2008.

[RS80] Michael Reed and Barry Simon, Methods of modern mathematical physics., second ed., Academic Press Inc. [Harcourt Brace Jovanovich Publishers], New York, 1980, Functional analysis. MR MR751959 (85e:46002)

[RT08] L. Rogers and A. Teplyaev, Laplacians on the Basilica julia set, preprint (2008).

[Rud91] Walter Rudin, Functional analysis, second ed., International Series in Pure and Applied Mathematics, McGraw-Hill Inc., New York, 1991. MR MR1157815 (92k:46001)

[Sab97] C. Sabot, Existence and uniqueness of diffusions on finitely ramified self-similar fractals, Ann. Sci. École Norm. Sup. (4) 30 (1997), no. 5, 605-673. MR MR1474807 (98h:60118)

[Sid98] Said N. Sidki, Regular trees and their automorphisms, Monografias de Matematica, vol. 56, IMPA, Rio de Janeiro, 1998.

[Sid00] , Automorphisms of one-rooted trees: growth, circuit structure and acyclicity, J. of Mathematical Sciences (New York) 100 (2000), no. 1, 1925-1943.

[Str06] Robert S. Strichartz, Differential equations on fractals, Princeton University Press, Princeton, NJ, 2006, A tutorial. MR MR2246975 (2007f:35003)

[Tep08] Alexander Teplyaev, Harmonic coordinates on fractals with finitely ramified cell structure, Canad. J. Math. (2008), to appear (CJM Pre-publication).

[VV07] Mariya Vorobets and Yaroslav Vorobets, On a free group of transformations defined by an automaton, Geometriae Dedicata 124 (2007), no. 1, 237-249.

Texas A\&M University, College Station

E-mail address: nekrash@math.tamu.edu

Department of Mathematics, University of Connecticut, Storrs CT 06269, USA

E-mail address: teplyaev@math.uconn.edu 